\title{
SCALING LIMIT OF A DISCRETE PRION DYNAMICS MODEL*
}

\author{
MARIE DOUMIC ${ }^{\dagger}$, THIERRY GOUDON ${ }^{\ddagger}$, AND THOMAS LEPOUTRE ${ }^{\S}$
}

\begin{abstract}
This paper investigates the connection between discrete and continuous models describing prion proliferation. The scaling parameters are interpreted on biological grounds and we establish rigorous convergence statements. We also discuss, based on the asymptotic analysis, relevant boundary conditions that can be used to complete the continuous model.
\end{abstract}

Key words. Aggregation fragmentation equations, asymptotic analysis, polymerization process.

AMS subject classifications. 35B45, 45K05, 92D25.

\section{Introduction}

The modelling of intracellular prion infection has been dramatically improved in the past few years according to recent progress in molecular biology of this pathology. Relevant models have been designed to investigate the conversion of the normal monomeric form of the protein (denoted $\mathrm{PrPc}$ ) into the infectious polymeric form (denoted PrPsc) according to the auto-catalytic process:

$$
\operatorname{PrPc}+\operatorname{PrPsc} \longrightarrow 2 \mathrm{PrPsc}
$$

in fibrillar aggregation of the protein. These models are based on linear growth of PrPsc polymers via an autocatalytic process [9].

The seminal paper by Masel et al. [17] proposed a discrete model where the prion population is described by its distribution with respect to the size of polymer aggregates. The model is an infinite-dimensional system of Ordinary Differential Equations, taking into account nucleated transconformation and polymerization, fragmentation and degradation of the polymers, as well as production of $\operatorname{PrPc}$ by the cells. This model consists in an aggregation fragmentation discrete model. In full generality, it writes as follows:

$$
\left\{\begin{array}{l}
\frac{\mathrm{d} v}{\mathrm{~d} t}=\lambda-\gamma v-v \sum_{i=n_{0}}^{\infty} \tau_{i} u_{i}+2 \sum_{j \geq n_{0}} \sum_{i<n_{0}} i k_{i, j} \beta_{j} u_{j}, \\
\frac{\mathrm{d} u_{i}}{\mathrm{~d} t}=-\mu_{i} u_{i}-\beta_{i} u_{i}-v\left(\tau_{i} u_{i}-\tau_{i-1} u_{i-1}\right)+2 \sum_{j>i} \beta_{j} k_{i, j} u_{j}, \quad \text { for } i \geq n_{0},
\end{array}\right.
$$

(with the convention $\tau_{n_{0}-1} u_{n_{0}-1}=0$ ). Here $v$ represents the quantity of healthy monomers (PrPc), $u_{i}$ the quantity of infectious polymers (PrPsc) of size $i$, i.e. formed by the fibrillar aggregation of $i$ monomers. We thus have $i \geqslant n_{0} \geqslant 2$, where $n_{0}$ represents the minimal size for polymers: smaller polymers are considered to be unstable

${ }^{*}$ Received: July 8, 2009; accepted (in revised version): September 19, 2009. Communicated by Benoit Perthame.

${ }^{\dagger}$ INRIA Research Centre Paris-Rocquencourt, Project-Team BANG, Domaine de Voluceau, BP 105, 781153 Rocquencourt, France (marie.doumic@inria.fr).

$\ddagger$ INRIA Research Centre Lille-Nord Europe, Project-Team SIMPAF, Park Plazza, 40 avenue Halley, F-Villeneuve d'Ascq cedex, France \& Labo. Paul Painlevé, UMR CNRS 8524, Université des Sciences et Technologies de Lille, (thierry.goudon@inria.fr).

§UPMC Univ. Paris 06, UMR 7598, Laboratoire Jacques-Louis Lions, F-75005, Paris, France \& CNRS, UMR 7598, Laboratoire Jacques-Louis Lions, F-75005, Paris, France \& INRIA Research Centre Paris-Rocquencourt, Project-Team BANG, (thomas.lepoutre@inria.fr). 
and are immediately degraded into monomers, as the last term of equation (1.1) for $v$ expresses. Parameters $\gamma$ and $\mu_{i}$ are the degradation rates respectively of monomers and polymers of size $i$. The parameter $\lambda$ is a source term: the basal synthesis rate of PrPc. The coefficient $\beta_{i}$ is the fragmentation rate of a polymer of size $i$, and the coefficient $k_{j, i}$ is the repartition function for a polymer of size $i$ dividing into two polymers of smaller sizes $j$ and $i-j$. Finally, the quantity $v \tau_{i}$ is the aggregation speed of polymers of size $i$, which is supposed to depend both on the available quantity of monomers $v$ and on a specific aggregation ability $\tau_{i}$ of polymers of size $i$.

In the original model [17], the degradation rate of polymers $\mu_{i}$ and the aggregation rate $\tau_{i}$ were assumed to be independent of the size $i$, the fragmentation rate satisfied $\beta_{j}=\beta(j-1)$ for a constant $\beta$ and $k_{i, j}$ was a uniform repartition over $\{1, \ldots j-1\}$, i.e., $k_{i, j}=\frac{1}{j-1}$ for $i \in\{1, \ldots j-1\}$, and 0 elsewhere. These laws express that all polymers behave in the same way, and that any joint point of any polymer has the same probability to break. It allowed the authors to close the system into an ODE system of three equations, which is quite simple to analyze. However, following recent experimental results such as in [20], and their mathematical analysis in [5, 4], here we prefer to consider variable coefficients in their full generality. Following the ideas of [7], we can consider, under reasonable growth assumptions on the coefficients, the so called admissible solutions, i.e., solutions obtained by taking the limit of truncated systems (see Appendix C).

Recent work by Greer et al. analyzed this process in a continuous setting [10]. They proposed a Partial Differential Equation to render out the above-mentioned polymerization/fragmentation process. It writes

$$
\begin{aligned}
\frac{\mathrm{d} V}{\mathrm{~d} t}= & \lambda-\gamma V-V \int_{x_{0}}^{\infty} \tau(x) U(t, x) \mathrm{d} x \\
& +2 \int_{x=x_{0}}^{\infty} \int_{y=0}^{x_{0}} y k(y, x) \beta(x) U(t, x) \mathrm{d} x \mathrm{~d} y, \\
\frac{\partial U}{\partial t}= & -\mu(x) U(t, x)-\beta(x) U(t, x)-V \frac{\partial}{\partial x}(\tau U) \\
& +2 \int_{x}^{\infty} \beta(y) k(x, y) U(t, y) \mathrm{d} y .
\end{aligned}
$$

The coefficients of the continuous model (1.2) (1.3) have the same meaning as those of the discrete one (1.1); however, some questions about their scaling remain, and in particular about the exact biological interpretation of the variable $x$.

The aim of this article is to investigate the link between system (1.1) and system (1.2), (1.3). We discuss in details the convenient mathematical assumptions under which we can ensure that the continuous system is the limit of the discrete one and we rigorously establish the convergence statement. We also want to discuss possible biological interpretations of our asymptotic analysis, and see how our work can help to define a proper boundary condition at $x=x_{0}$ for system (1.2), (1.3). Indeed, equation (1.3) holds in the domain $x>x_{0}$ and, due to the convection term, at least when $V(t) \tau\left(x_{0}\right)>0$ a boundary condition is necessary to complete the problem.

In section 2, we first recall general properties and previous results on the considered equations. In section 3, we rescale the equations in order to make a small parameter $\varepsilon$ appear, and we state the main result: the asymptotic convergence of the 
rescaled discrete system towards the continuous equations. Section 4 is devoted to its proof, based on moments a priori estimates. Sections 5 and 6 discuss how these results can be interpreted on physical grounds. We also comment the issue of the boundary condition for the continuous model.

\section{Basic properties of the equations}

All the considered coefficients are nonnegative. We need some structural hypotheses on $k$ and $k_{j, i}$ to make sense. Obviously, the hypotheses take into account that a polymer can only break into smaller pieces. We also impose symmetry since a given polymer of size $y$ breaks equally into two polymers of size $x$ and $y-x$, respectively. Summarizing, we have

$$
\begin{array}{ll}
k_{i, j} \geq 0, & k(x, y) \geq 0, \\
k_{i, j}=0 \text { for } i \geq j & k(x, y)=0 \text { for } x>y, \\
k_{i, j}=k_{j-i, i}, & k(x, y)=k(y-x, y), \\
\sum_{i=1}^{j-1} k_{i, j}=1, & \int_{0}^{y} k(x, y) \mathrm{d} x=1 .
\end{array}
$$

(Note that $(2.1)$ and $(2.3)$ imply that $0 \leq k_{i, j} \leq 1$.) Classically, these two conditions lead to a third one, expressing mass conservation through the fragmentation process:

$$
2 \sum_{i=1}^{j-1} i k_{i, j}=j, \quad 2 \int_{0}^{y} x k(x, y) \mathrm{d} x=y .
$$

The discrete equation belongs to the family of coagulation-fragmentation models (see $[1,2])$. Adapting the work of $[1,2]$ to this system, we obtain the following result. It is not optimal but sufficient for our study.

THEOREM 2.1. Let $k_{i . j}$ satisfy Assumptions (2.1)-(2.3). We assume the following growth estimate on the coefficients: there exist $K>0, \alpha \geq 0, m \geq 0$, and $0 \leq \theta \leq 1$ such that

$$
0 \leq \beta_{i} \leq K i^{\alpha}, \quad 0 \leq \mu_{i} \leq K i^{m}, \quad 0 \leq \tau_{i} \leq K i^{\theta} .
$$

The initial data $v^{0} \geq 0, u_{i}^{0} \geq 0$ satisfies, for $\sigma=\max (1+m, 1+\theta, \alpha)$

$$
\sum_{i=n_{0}}^{\infty} i^{\sigma} u_{i}^{0}<+\infty
$$

Then there exists a unique global solution to (1.1) which satisfies for all $t \geq 0$

$$
v(t)+\sum_{i=n_{0}}^{\infty} i u_{i}(t)=v^{0}+\sum_{i=n_{0}}^{\infty} i u_{i}^{0}+\lambda t-\int_{0}^{t} \gamma v(s) \mathrm{d} s-\int_{0}^{t} \sum_{i=n_{0}}^{\infty} i \mu_{i} u_{i}(s) \mathrm{d} s .
$$

A sketch of the proof is given in Appendix C. Let us introduce the quantity

$$
\rho(t)=v(t)+\sum_{i=n_{0}}^{\infty} i u_{i}(t)
$$


which is the total number of monomers in the population. Equation (2.6) is a mass balance equation, which can be written as

$$
\frac{\mathrm{d}}{\mathrm{d} t} \rho=\lambda-\gamma v(t)-\sum_{i=n_{0}}^{\infty} i \mu_{i} u_{i}(t) .
$$

Similarly for the continuous model we define

$$
\varrho(t)=V(t)+\int_{x_{0}}^{\infty} x U(t, x) \mathrm{d} x .
$$

The analogue of (2.8) would be

$$
\varrho(t)-\varrho(0)=\lambda t-\int_{0}^{t} \gamma V(s) \mathrm{d} s-\int_{0}^{t} \int_{x_{0}}^{\infty} x \mu(x) U(t, x) \mathrm{d} x .
$$

In fact, the argument to deduce (2.9) from the system (1.2)(1.3) is two-fold: it relies both on the boundary condition on $\left\{x=x_{0}\right\}$ for (1.3) and on the integrability properties of the fragmentation term

$$
x \times\left(2 \int_{x}^{\infty} \beta(y) k(x, y) U(t, y) \mathrm{d} y-\beta(x) U(t, x)\right),
$$

the integral of which has to be combined to (1.2) by virtue of (2.4). The question is actually quite deep, as it is already revealed by the case where $\mu=0, \tau=0$ and $x_{0}=0$. In this situation it can be shown that (1.3) admits solutions that do not satisfy the conservation law: $\int_{0}^{\infty} x U(t, x) \mathrm{d} x=\int_{0}^{\infty} x U(0, x) \mathrm{d} x$, see [8]. Hence, (2.9) has to be incorporated in the model as a constraint to select the physically relevant solution, as suggested in [8] and [13]. Nevertheless, the integrability of the fragmentation term is not a big deal since it can be obtained by imposing boundedness of a large enough moment of the initial data as it will be clear in the discussion below and as it appeared in $[8,13]$. More interesting is how to interpret this in terms of boundary conditions; we shall discuss the point in section 5 . (Note that in [13] the problem is completed with the boundary condition $U\left(t, x_{0}\right)=0$ while $x_{0}>0, \tau\left(x_{0}\right)>0$.) According to $[8,13]$ we adopt the following definition.

DEFINITION 2.2. We say that the pair $(U, V)$ is a "monomer preserving weak solution of the prion proliferation equations" with initial data $\left(U_{0}, V_{0}\right)$ if it satisfies $(1.2)$ and if for any $\varphi \in \mathcal{C}_{c}^{\infty}\left(\left(x_{0}, \infty\right)\right)$, we have

$$
\begin{aligned}
& \int_{0}^{\infty} U(t, \mathrm{~d} x) \varphi(x)-\int_{0}^{\infty} U_{0}(\mathrm{~d} x) \varphi(x) \\
=-\int_{0}^{t} & \int_{0}^{\infty} \mu(x) U(s, \mathrm{~d} x) \varphi(x) \mathrm{d} s-\int_{0}^{t} \int_{0}^{\infty} \beta(x) U(s, \mathrm{~d} x) \varphi(x) \mathrm{d} s \\
& +\int_{0}^{t} V(s) \int_{0}^{\infty} \tau(x) U(s, \mathrm{~d} x) \partial_{x} \varphi(x) \mathrm{d} s \\
& +2 \int_{0}^{t} \int_{x_{0}}^{\infty} \beta(y) U(s, \mathrm{~d} y) \int_{x_{0}}^{y} k(\mathrm{~d} x, y) \varphi(x) \mathrm{d} s,
\end{aligned}
$$

and

$$
\begin{aligned}
V(t)+\int_{x_{0}}^{\infty} x U(t, \mathrm{~d} x)= & V_{0}+\int_{x_{0}}^{\infty} x U_{0}(\mathrm{~d} x) \\
& +\lambda t-\int_{0}^{t} \gamma V(s) \mathrm{d} s-\int_{0}^{t} \int_{x_{0}}^{\infty} x \mu(x) U(s, \mathrm{~d} x) \mathrm{d} s
\end{aligned}
$$


A break is necessary to discuss the functional framework to be used in Definition 2.2. We start by setting up some notation. We denote $\mathcal{M}^{1}(X)$ to be the set of bounded Radon measures on a Borelian set $X \subset \mathbb{R} ; \mathcal{M}_{+}^{1}(X)$ stands for the positive cone in $\mathcal{M}^{1}(X)$. The space $\mathcal{M}^{1}(X)$ identifies as the dual of the space $\mathcal{C}_{0}(X)$ of continuous functions vanishing at infinity in $X,{ }^{1}$ endowed with the supremum norm, see [15]. Given an interval $I \subset \mathbb{R}$, we consider measure valued functions $W: y \in I \mapsto W(y) \in$ $\mathcal{M}^{1}(X)$. Denoting $W(y, \mathrm{~d} x)=W(y)(\mathrm{d} x)$, we say that $W \in \mathcal{C}\left(I ; \mathcal{M}^{1}(X)-\right.$ weak $\left.-\star\right)$, if, for any $\varphi \in \mathcal{C}_{0}(X)$, the function $y \mapsto \int_{X} \varphi(x) W(y, \mathrm{~d} x)$ is continuous on $I$. We are thus led to assume

$$
U \in \mathcal{C}\left([0, T] ; \mathcal{M}_{+}^{1}([0, \infty))-\text { weak }-\star\right), \quad V \in \mathcal{C}([0, T]),
$$

with furthermore

$$
\operatorname{supp}(U(t, .)) \subset\left[x_{0}, \infty\right), \quad \int_{x_{0}}^{\infty} x U(t, \mathrm{~d} x)<\infty,
$$

which corresponds to the physical meaning of the unknowns. Hence, formula (2.10) makes sense for continuous coefficients

$$
\mu, \beta, \tau \in \mathcal{C}\left(\left[x_{0}, \infty\right)\right)
$$

Concerning the fragmentation kernel, it suffices to suppose that

$$
y \mapsto k(\mathrm{~d} x, y) \in \mathcal{C}\left(\left[x_{0}, \infty\right) ; \mathcal{M}_{+}^{1}([0, \infty))-\text { weak }-\star\right) .
$$

\section{Main result}

This section splits into three steps: firstly, we precisely set up the scaling of the equations, secondly we detail the assumptions on the discrete coefficients which will be used to define, through a compactness argument, the coefficients of the limit equations, and thirdly we give the main statement of the paper.

3.1. Notations and rescaled equations. We first rewrite system (1.1) in a dimensionless form, as done for instance in [6] (see also [11]). Here we summarize all the absolute constants that we will need in the sequel:

- $T$, characteristic time,

- $\mathcal{U}$, characteristic value for the concentration of polymers $u_{i}$,

- $\mathcal{V}$, characteristic value for the concentration of monomers $v$,

- $\mathcal{T}$, characteristic value for the polymerisation rate $\tau_{i}$,

- $B$, characteristic value for the fragmentation frequency $\beta_{i}$,

- $d_{0}$, characteristic value for the degradation frequency of polymers $\mu_{i}$,

- $\Gamma$, characteristic value for the degradation frequency of monomers $\gamma$,

- $L$, characteristic value for the source term $\lambda$.

\footnotetext{
${ }^{1} \phi \in \mathcal{C}_{0}(X)$ means that $\phi$ is continuous and for any $\eta>0$, there exists a compact set $K \subset X$ such that $\sup _{X \backslash K}|\phi(x)| \leq \eta$. We denote $\mathcal{C}_{c}(X)$ to be the space of continuous functions with compact support in $X$.
} 
The dimensionless quantities are defined by

$$
\begin{array}{lll}
\bar{t}=\frac{t}{T}, & \bar{v}(\bar{t})=\frac{v(\bar{t} T)}{\mathcal{V}}, & \bar{u}_{i}(\bar{t})=\frac{u_{i}(\bar{t} T)}{\mathcal{U}}, \quad \bar{\beta}_{i}=\frac{\beta_{i}}{B}, \quad \bar{\tau}_{i}=\frac{\tau_{i}}{\mathcal{T}}, \\
\bar{\mu}_{i}=\frac{\mu_{i}}{d_{0}}, \quad \bar{\lambda}=\frac{\lambda}{L}, & \bar{\gamma}=\frac{\gamma}{\Gamma} .
\end{array}
$$

Recall that $k_{i, j}$ is already dimensionless. The following dimensionless parameters arise from this nondimensionalization

$$
\left\{\begin{array}{l}
a=\frac{L T}{\mathcal{V}}, b=B T, \quad c=\Gamma T, d=d_{0} T \\
s=\frac{\mathcal{U}}{\mathcal{V}}, \quad \nu=T \mathcal{T} \mathcal{V}
\end{array}\right.
$$

Omitting the overlines, the equation becomes

$$
\left\{\begin{array}{l}
\frac{\mathrm{d} v}{\mathrm{~d} t}=a \lambda-c \gamma v-\nu s v \sum \tau_{i} u_{i}+2 b s \sum_{j \geq n_{0}} \sum_{i<n_{0}} i k_{i, j} \beta_{j} u_{j}, \\
\frac{\mathrm{d} u_{i}}{\mathrm{~d} t}=-d \mu_{i} u_{i}-b \beta_{i} u_{i}-\nu v\left(\tau_{i} u_{i}-\tau_{i-1} u_{i-1}\right)+2 b \sum_{j>i} \beta_{j} k_{i, j} u_{j}, \quad \text { for } i \geq n_{0} .
\end{array}\right.
$$

The definition (2.7) of the total mass in dimensionless form becomes

$$
v+s \sum_{i=n_{0}}^{\infty} i u_{i}=\rho .
$$

The rationale motivating the scaling can be explained as follows. Let $0<\varepsilon \ll 1$ be a parameter intended to tend to 0 . We pass from the discrete model to the continuous model by associating a stepwise constant function to the $u_{i}$ 's, constant on each interval $(\varepsilon i, \varepsilon(i+1))$. Then sums over the index $i$ will be interpreted as Riemann sums which are expected to tend to integrals in the continuum limit while finite differences will give rise to derivatives. Having in mind the case of homogeneous division and polymerization rates $\beta(x)=x^{\alpha}, \tau(x)=x^{\theta}, \mu(x)=x^{m}$, which generalizes the constantcoefficient case proposed by [10], and their discrete analogue $\beta_{i}=i^{\alpha}, \tau_{i}=i^{\theta}, \mu_{i}=i^{m}$, we shall assume that the rescaled coefficients $\beta_{i}, \mu_{i}, \tau_{i}$ fulfill (2.5). Therefore, we are led to set

$$
s=\varepsilon^{2}
$$

so that (3.3) becomes

$$
v+\varepsilon \sum_{i=n_{0}}^{\infty} \varepsilon i u_{i}=\rho,
$$

to be compared to the definition of $\varrho$ in $(2.9)$. This scaling means that the typical concentration of any aggregate with size $i>n_{0}$ is small compared to the monomers concentration, but the total mass of the aggregates is in the order of the mass of monomers. Next, we set

$$
a=1, \quad b=\varepsilon^{\alpha}, \quad c=1, \quad d=\varepsilon^{m}, \quad \nu=\varepsilon^{\theta-1} .
$$


The rescaled equations read

$$
\left\{\begin{array}{l}
\frac{\mathrm{d} v}{\mathrm{~d} t}=\lambda-\gamma v-\varepsilon^{\theta+1} v \sum \tau_{i} u_{i}+2 \varepsilon^{2+\alpha} \sum_{i \geq n_{0}} \sum_{j<n_{0}} j k_{j, i} \beta_{i} u_{i}, \\
\frac{\mathrm{d} u_{i}}{\mathrm{~d} t}=-\varepsilon^{m} \mu_{i} u_{i}-\varepsilon^{\alpha} \beta_{i} u_{i}-\varepsilon^{\theta-1} v\left(\tau_{i} u_{i}-\tau_{i-1} u_{i-1}\right)+2 \varepsilon^{\alpha} \sum_{j>i} \beta_{j} k_{i, j} u_{j}, \quad \text { for } i \geq n_{0} .
\end{array}\right.
$$

Eventually, the threshold value $n_{0}$ also depends on the scaling parameter and we assume

$$
\lim _{\varepsilon \rightarrow 0} \varepsilon n_{0}(\varepsilon)=x_{0} \geq 0 .
$$

This choice is discussed in section 6.3 .

Equation (3.5) is completed by an initial data $\left(u_{i}^{0, \varepsilon}, v^{0, \varepsilon}\right)$ verifying, for some constants $M_{0}, \rho^{0}, M_{1+\sigma}$ independent of $\varepsilon$ :

$$
\left\{\begin{array}{l}
v^{0, \varepsilon}+\varepsilon^{2} \sum_{i=n_{0}(\varepsilon)}^{\infty} i u_{i}^{0, \varepsilon}=\rho^{0}<+\infty, \\
\varepsilon \sum_{i=n_{0}(\varepsilon)}^{\infty} u_{i}^{0, \varepsilon} \leq M_{0}<+\infty, \\
\varepsilon^{2+\sigma} \sum_{i=n_{0}(\varepsilon)}^{\infty} i^{1+\sigma} u_{i}^{0, \varepsilon} \leq M_{1+\sigma}<+\infty, \quad 1+\sigma>\max (1, \alpha, 1+m, 1+\theta) .
\end{array}\right.
$$

For any $0<T<\infty$, Theorem 2.1 guarantees the existence of a solution $\left(u_{i}^{\varepsilon}, v^{\varepsilon}\right)$ of $(3.5)$. Let us set

$$
\chi_{i}^{\varepsilon}(x)=\chi_{[i \varepsilon,(i+1) \varepsilon)}(x),
$$

with $\chi_{A}$ the indicator function of a set $A$. We introduce the piecewise constant function

$$
u^{\varepsilon}(t, x):=\sum_{i=n_{0}(\varepsilon)}^{\infty} u_{i}^{\varepsilon}(t) \chi_{i}^{\varepsilon}(x) .
$$

On the same token, we associate the following functions to the coefficients

$$
\begin{aligned}
k^{\varepsilon}(x, y) & :=\sum_{i=0}^{\infty} \sum_{j=0}^{\infty} \frac{k_{i, j}}{\varepsilon} \chi_{i}^{\varepsilon}(x) \chi_{j}^{\varepsilon}(y), \\
\mu^{\varepsilon}(x) & :=\sum_{i=n_{0}(\varepsilon)}^{\infty} \varepsilon^{m} \mu_{i} \chi_{i}^{\varepsilon}(x), \\
\beta^{\varepsilon}(x) & :=\sum_{i=n_{0}(\varepsilon)}^{\infty} \varepsilon^{\alpha} \beta_{i} \chi_{i}^{\varepsilon}(x), \\
\tau^{\varepsilon}(x) & :=\sum_{i=n_{0}(\varepsilon)}^{\infty} \varepsilon^{\theta} \tau_{i} \chi_{i}^{\varepsilon}(x) .
\end{aligned}
$$

This choice is made so that for all $y, k^{\varepsilon}(\cdot, y)$ is a probability measure on $[0, y]$. 
3.2. Compactness assumptions on the coefficients. For technical purposes we need further assumptions on the discrete coefficients. Let us collect them as follows: there exists $K>0$ such that

$$
\begin{aligned}
& \left|\beta_{i+1}-\beta_{i}\right| \leq K i^{\alpha-1} \\
& \left|\mu_{i+1}-\mu_{i}\right| \leq K i^{m-1} \\
& \left|\tau_{i+1}-\tau_{i}\right| \leq K i^{\theta-1}
\end{aligned}
$$

where the exponents $\alpha, \theta, m$ are defined in (2.5). For the fragmentation kernel we furthermore assume that there exists $K>0$ such that for any $i, j$

$$
\left|\sum_{p=0}^{i-1} \sum_{r=0}^{p-1} k_{r, j+1}-\sum_{p=0}^{i-1} \sum_{r=0}^{p-1} k_{r, j}\right| \leq K
$$

These assumptions will be helpful for investigating the behavior of (3.5) as $\varepsilon$ goes to 0 since they provide compactness properties. We summarize these properties in the following lemma.

LEMma 3.1. Let $\left(z_{i}\right)_{i \in \mathbb{N}}$ be a sequence of nonnegative real numbers verifying

$$
0 \leq z_{i} \leq K i^{\kappa}, \quad\left|z_{i+1}-z_{i}\right| \leq K i^{\kappa-1}
$$

for some $K>0$ and $\kappa \geq 0$. For $x \geq 0$, we set $z^{\varepsilon}(x)=\sum_{i} \varepsilon^{\kappa} z_{i} \chi_{[\varepsilon i, \varepsilon(i+1))}(x)$. Then there exists a subsequence $\varepsilon_{n} \rightarrow 0$, and a continuous function $z: x \in[0, \infty) \mapsto z(x)$ such that $z^{\varepsilon_{n}}$ converges to $z$ uniformly on $[r, R]$ for any $0<r<R<\infty$. If $\kappa>0$, the convergence holds on $[0, R]$ for any $0<R<\infty$ and we have $z(0)=0$.

We shall apply this statement to the sequences $\beta^{\varepsilon}, \mu^{\varepsilon}, \tau^{\varepsilon}$. A similar compactness property can be obtained for the fragmentation coefficients.

LEMma 3.2. Let the coefficients $k_{i, j}$ satisfy Assumptions (2.2),(2.3), and (3.9). Then there exists a subsequence $\left(\varepsilon_{n}\right)_{n \in \mathbb{N}}$ and $k: y \in[0, \infty) \mapsto k(\mathrm{~d} x, y) \in \mathcal{M}_{+}^{1}([0, \infty))$ which belongs to $\mathcal{C}\left([0, \infty) ; \mathcal{M}_{+}^{1}([0, \infty))\right.$ - weak $\left.-\star\right)$ satisfying also (2.2) and (2.3) (in their continuous version) and such that $k^{\varepsilon_{n}}$ converges to $k$ in the following sense: for every compactly supported smooth function $\varphi \in \mathcal{C}_{c}^{\infty}\left(\left[x_{0}, \infty\right)\right)$, denoting

$$
\phi^{\varepsilon_{n}}(y)=\int_{n_{0}\left(\varepsilon_{n}\right) \varepsilon_{n}}^{y} k^{\varepsilon_{n}}(x, y) \varphi(x) \mathrm{d} x, \quad \phi(y)=\int_{x_{0}}^{y} k(\mathrm{~d} x, y) \varphi(x),
$$

we have $\phi^{\varepsilon_{n}} \rightarrow \phi$ uniformly locally in $\left[x_{0},+\infty\right)$.

The detailed proofs of Lemma 3.1 and Lemma 3.2 are postponed to Appendix B.

3.3. Main results. We are now ready to state the main results of this article. THEOREM 3.3. Assume (2.5) and (3.8). Suppose the fragmentation coefficients fulfill (2.1)-(2.3) and (3.9). Then, there exist a subsequence, denoted $\left(\varepsilon_{n}\right)_{n \in \mathbb{N}}$, continuous functions $\mu, \tau, \beta$, and a nonnegative measure-valued function $k(\mathrm{~d} x, y)$ verifying (2.2) and (2.3), such that

$$
\mu^{\varepsilon_{n}}, \tau^{\varepsilon_{n}}, \beta^{\varepsilon_{n}}, k^{\varepsilon_{n}} \rightarrow \mu, \tau, \beta, k
$$

in the sense of Lemma 3.1 and Lemma 3.2. 
Let the initial data satisfy (3.7). Then we can choose the subsequence $\left(\varepsilon_{n}\right)_{n \in \mathbb{N}}$ such that there exists $(U, V)$ for which

$$
\left\{\begin{array}{l}
\left.u^{\varepsilon_{n}} \rightarrow U, \text { in } \mathcal{C}\left([0, T] ; \mathcal{M}^{1}([0, \infty))-\text { weak }-\star\right)\right), \\
v^{\varepsilon_{n}}-V \quad \text { uniformly on }[0, T] .
\end{array}\right.
$$

We have $x U(t, \mathrm{~d} x) \in \mathcal{M}^{1}([0, \infty))$, the measure $U(t, \mathrm{~d} x)$ has its support included in $\left[x_{0},+\infty\right)$ for all time $t \geq 0$, and $(U, V)$ satisfies (2.10)-(2.11).

TheOREM 3.4. The limit $(U, V)$ exhibited in Theorem 3.3 is a monomer preserving weak solution (i.e., also satisfies equation (1.2)) in the following situations:

i) $x_{0}=0$ and either $\theta>0$ (so that the limit $\tau$ satisfies $\tau(0)=0$ ), or the rates $\tau_{i}=\tau$ are constant.

ii) $x_{0}>0$ and the discrete fragmentation coefficients fulfill the following strengthened assumption: for any $i, j$ we have

$$
\left|\sum_{i^{\prime} \leq i}\left(k_{i^{\prime} j+1}-k_{i^{\prime}, j}\right)\right| \leq \frac{K}{j}, \quad k_{i, j} \leq \frac{K}{j} .
$$

\section{Moment estimates}

We start by establishing a priori estimates uniformly with respect to $\varepsilon$. These estimates will induce compactness properties on the sequence of solutions. As described in [12] for general coagulation fragmentation models, the model has the property of propagating moments.

Lemma 4.1. Let the assumptions of Theorem 3.3 be fulfilled. Then for any $T>0$, there exists a constant $C<\infty$ which only depends on $M_{0}, M_{1+\sigma}, \rho^{0}, \lambda, K$ and $T$, such that for any $\varepsilon>0$,

$$
\sup _{t \in[0, T]} \int_{0}^{\infty}\left(1+x+x^{1+\sigma}\right) u^{\varepsilon}(t, x) \mathrm{d} x \leq C, \quad 0 \leq v^{\varepsilon}(t) \leq C .
$$

Proof. For $r \geq 0$, we denote

$$
M_{r}^{\varepsilon}(t)=\varepsilon \sum_{i=n_{0}}^{\infty}(i \varepsilon)^{r} u_{i}^{\varepsilon}(t) .
$$

As in [6], we can notice that

$$
\int_{0}^{\infty}\left(\frac{x}{2}\right)^{r} u^{\varepsilon}(t, x) \mathrm{d} x \leq M_{r}^{\varepsilon}(t) \leq \int_{0}^{\infty} x^{r} u^{\varepsilon}(t, x) \mathrm{d} x .
$$

Therefore, we only need to control $M_{0}^{\varepsilon}(t), M_{1}^{\varepsilon}(t)$ and $M_{1+\sigma}^{\varepsilon}(t)$. We notice the obvious but useful inequality, for $0 \leq r \leq 1+\sigma$,

$$
(i \varepsilon)^{r} \leq 1+(i \varepsilon)^{1+\sigma},
$$

and therefore,

$$
\left|M_{r}^{\varepsilon}\right| \leq\left|M_{0}^{\varepsilon}\right|+\left|M_{1+\sigma}^{\varepsilon}\right|
$$


In the sequel, we use alternatively two equivalent discrete weak formulations of equation (3.5) in the spirit of [13]. We multiply the second equation of (3.5) by $\varphi_{i}$ and summing over $i$, we first obtain

$$
\begin{aligned}
\frac{\mathrm{d}}{\mathrm{d} t} \sum_{i=n_{0}}^{\infty} u_{i}^{\varepsilon} \varphi_{i}= & -\varepsilon^{m} \sum_{i=n_{0}}^{\infty} \mu_{i} u_{i}^{\varepsilon} \varphi_{i}-\varepsilon^{\alpha} \sum_{i=n_{0}}^{\infty} \beta_{i} u_{i}^{\varepsilon} \varphi_{i} \\
& -\varepsilon^{\theta-1} \sum_{i=n_{0}}^{\infty} v^{\varepsilon}\left(\tau_{i} u_{i}^{\varepsilon}-\tau_{i-1} u_{i-1}^{\varepsilon}\right) \varphi_{i}+2 \varepsilon^{\alpha} \sum_{i=n_{0}}^{\infty} \varphi_{i} \sum_{j>i} \beta_{j} k_{i, j} u_{j}^{\varepsilon}, \\
= & -\varepsilon^{m} \sum_{i=n_{0}}^{\infty} \mu_{i} u_{i}^{\varepsilon} \varphi_{i}-\varepsilon^{\alpha} \sum_{i=n_{0}}^{\infty} \beta_{i} u_{i}^{\varepsilon} \varphi_{i}+\varepsilon^{\theta-1} v^{\varepsilon} \sum_{i=n_{0}}^{\infty} \tau_{i} u_{i}^{\varepsilon}\left(\varphi_{i+1}-\varphi_{i}\right) \\
& +2 \varepsilon^{\alpha} \sum_{i=n_{0}}^{\infty} \varphi_{i} \sum_{j>i} \beta_{j} k_{i, j} u_{j}^{\varepsilon}
\end{aligned}
$$

Using the properties of $k_{i, j}$, we rewrite the fragmentation terms as follows

$$
\begin{aligned}
\sum_{i=n_{0}}^{\infty} \beta_{i} u_{i}^{\varepsilon} \varphi_{i} & =2 \sum_{j=n_{0}+1}^{\infty} \beta_{j} \sum_{i=1}^{j-1} i k_{i, j} u_{j}^{\varepsilon} \frac{\varphi_{j}}{j}+\beta_{n_{0}} u_{n_{0}}^{\varepsilon} \varphi_{n_{0}} \\
& =2 \sum_{j=n_{0}+1}^{\infty} \sum_{i=n_{0}}^{j-1} i k_{i, j} \beta_{j} u_{j}^{\varepsilon} \frac{\varphi_{j}}{j}+2 \sum_{j=n_{0}+1}^{\infty} \sum_{i=1}^{n_{0}-1} i k_{i, j} \beta_{j} u_{j}^{\varepsilon} \frac{\varphi_{j}}{j}+\beta_{n_{0}} u_{n_{0}}^{\varepsilon} \varphi_{n_{0}}, \\
2 \sum_{i=n_{0}}^{\infty} \varphi_{i} \sum_{j>i} \beta_{j} k_{i, j} u_{j}^{\varepsilon} & =2 \sum_{j=n_{0}+1}^{\infty} \sum_{i=n_{0}}^{j-1} i k_{i, j} \beta_{j} u_{j}^{\varepsilon} \frac{\varphi_{i}}{i} .
\end{aligned}
$$

By using (2.4), we have $n_{0}=2 \sum_{i=1}^{n_{0}-1} i k_{i, n_{0}}$ and we obtain

$$
\begin{aligned}
2 \sum_{i=n_{0}}^{\infty} \varphi_{i} \sum_{j>i} \beta_{j} k_{i, j} u_{j}^{\varepsilon}-\sum_{i=n_{0}}^{\infty} \beta_{i} u_{i}^{\varepsilon} \varphi_{i}= & -2 \sum_{j=n_{0}}^{\infty} \sum_{i=1}^{n_{0}-1} i k_{i, j} \beta_{j} u_{j}^{\varepsilon} \frac{\varphi_{j}}{j} \\
& +2 \sum_{j=n_{0}+1}^{\infty} \sum_{i=n_{0}}^{j-1} i k_{i, j} \beta_{j} u_{j}^{\varepsilon}\left(\frac{\varphi_{i}}{i}-\frac{\varphi_{j}}{j}\right) .
\end{aligned}
$$

Substituting this in the weak formulation we obtain

$$
\begin{aligned}
\frac{\mathrm{d}}{\mathrm{d} t} \sum_{i=n_{0}}^{\infty} u_{i}^{\varepsilon} \varphi_{i}= & -\varepsilon^{m} \sum_{i=n_{0}}^{\infty} \mu_{i} u_{i}^{\varepsilon} \varphi_{i}+\varepsilon^{\theta-1} v^{\varepsilon} \sum_{i=n_{0}}^{\infty} \tau_{i} u_{i}^{\varepsilon}\left(\varphi_{i+1}-\varphi_{i}\right) \\
& +2 \varepsilon^{\alpha} \sum_{j=n_{0}+1}^{\infty} \sum_{i=n_{0}}^{j-1} i k_{i, j} \beta_{j} u_{j}^{\varepsilon}\left(\frac{\varphi_{i}}{i}-\frac{\varphi_{j}}{j}\right)-2 \varepsilon^{\alpha} \sum_{j=n_{0}}^{\infty} \sum_{i=1}^{n_{0}-1} i k_{i, j} \beta_{j} u_{j}^{\varepsilon} \frac{\varphi_{j}}{j} .
\end{aligned}
$$

This last formulation makes the estimates straightforward (the computations are formal but can be understood as uniform bounds on solutions of truncated systems and therefore on any admissible solution). Taking $\phi_{i}=i \varepsilon$, we obtain the first moment, that is, the previously seen mass balance:

$$
\frac{\mathrm{d}}{\mathrm{d} t}\left(v^{\varepsilon}+\varepsilon^{2} \sum_{i=n_{0}}^{\infty} i u_{i}^{\varepsilon}\right)=-\gamma v^{\varepsilon}-\varepsilon^{2+m} \sum_{i=n_{0}}^{\infty} \mu_{i} i u_{i}^{\varepsilon}+\lambda \leq \lambda .
$$


Therefore, we get $\left(u_{i}^{\varepsilon}\right.$ and $v^{\varepsilon}$ are nonnegative)

$$
0 \leq v^{\varepsilon}(t)+M_{1}^{\varepsilon}(t) \leq \rho^{0}+\lambda T \quad \text { for } 0 \leq t \leq T<\infty,
$$

and

$$
\int_{0}^{t} \varepsilon^{2+m} \sum_{i=n_{0}}^{\infty} \mu_{i} i u_{i}^{\varepsilon}(s, x) \mathrm{d} s \leq \rho^{0}+\lambda T \quad \text { for } 0 \leq t \leq T<\infty .
$$

To obtain an estimate on the 0th order moment, we take $\varphi_{i}=\varepsilon$. The term with $\tau_{i}$ vanishes. Only considering the nonnegative part of the derivative, we derive from $(4.2)$

$$
\begin{aligned}
\frac{\mathrm{d}}{\mathrm{d} t} M_{0}^{\varepsilon}(t) & \leq 2 \varepsilon^{1+\alpha} \sum_{j=n_{0}+1}^{\infty} \sum_{i=n_{0}}^{j-1} i k_{i, j} \beta_{j} u_{j}^{\varepsilon} \frac{1}{i} \\
& \leq 2 \varepsilon^{1+\alpha} \sum_{j=n_{0}+1}^{\infty} \beta_{j} u_{j}^{\varepsilon} \leq 2 K M_{\alpha}^{\varepsilon}(t) .
\end{aligned}
$$

To give the bound on the $(1+\sigma)$ th moment, we choose $\varphi_{i}=\varepsilon(\varepsilon i)^{1+\sigma}$ in the weak formulation. Thanks to the mean value inequality, we have

$$
\left((\varepsilon(i+1))^{1+\sigma}-(\varepsilon i)^{1+\sigma}\right) \leq(1+\sigma) \varepsilon(\varepsilon(i+1))^{\sigma} \leq(1+\sigma) 2^{\sigma} \varepsilon(\varepsilon i)^{\sigma},
$$

therefore (4.2) yields

$$
\begin{aligned}
\frac{\mathrm{d}}{\mathrm{d} t} M_{1+\sigma}^{\varepsilon}(t)+\varepsilon^{1+m} \sum_{i=n_{0}}^{\infty} \mu_{i}(\varepsilon i)^{1+\sigma} u_{i}^{\varepsilon} & \leq \varepsilon^{\theta-1} v^{\varepsilon} \sum_{i=n_{0}}^{\infty} \tau_{i} u_{i}^{\varepsilon} \times \varepsilon \times\left((\varepsilon(i+1))^{1+\sigma}-(\varepsilon i)^{1+\sigma}\right) \\
& \leq v^{\varepsilon}(1+\sigma) 2^{\sigma} \sum_{i=n_{0}}^{\infty} \varepsilon^{\theta} \tau_{i} u_{i}^{\varepsilon} \varepsilon(\varepsilon i)^{\sigma} \\
& \leq K\left(\rho^{0}+\lambda T\right)(1+\sigma) 2^{\sigma} M_{\theta+\sigma}^{\varepsilon}(t) .
\end{aligned}
$$

Since $0 \leq \theta \leq 1$, and $1+\sigma>\alpha$ ( $\alpha$ is the exponent characterizing the growth of the fragmentation coefficient), denoting $C=\max \left(K\left(\rho^{0}+\lambda T\right)(1+\sigma) 2^{\sigma}, 2 K\right)$, we are led to

$$
\frac{\mathrm{d}}{\mathrm{d} t}\left(M_{0}^{\varepsilon}(t)+M_{1+\sigma}^{\varepsilon}(t)\right) \leq C\left(M_{\alpha}^{\varepsilon}(t)+M_{\theta+\sigma}^{\varepsilon}(t)\right) \leq 2 C\left(M_{0}^{\varepsilon}(t)+M_{1+\sigma}^{\varepsilon}(t)\right),
$$

and we conclude by the Gronwall lemma. It ends the proof of Lemma 4.1.

Hereafter, we denote $C$ to be a constant depending only on $T, M_{0}, \rho^{0}, M_{1+\sigma}, K$ and $\lambda$ such that

$$
M_{0}^{\varepsilon}, v^{\varepsilon}, M_{1}^{\varepsilon}, M_{1+\sigma}^{\varepsilon} \leq C .
$$

LEMMA 4.2. Under the assumptions of Lemma 4.1, the sequence of monomers concentration $\left(v^{\varepsilon}\right)_{\varepsilon>0}$ is equicontinuous on $[0, T]$.

Proof. We use the estimates of Lemma 4.1 to evaluate the derivative of $v^{\varepsilon}$. We recall the equation satisfied by $v^{\varepsilon}$

$$
\frac{\mathrm{d} v^{\varepsilon}}{\mathrm{d} t}=\lambda-\gamma v^{\varepsilon}+\varepsilon^{1+\theta} v^{\varepsilon} \sum \tau_{i} u_{i}^{\varepsilon}+2 \varepsilon^{2+\alpha} \sum_{i \geq n_{0}} \sum_{j<n_{0}} j k_{j, i} \beta_{i} u_{i}^{\varepsilon},
$$


which implies

$$
\left|\frac{\mathrm{d} v^{\varepsilon}}{\mathrm{d} t}\right| \leq \lambda+\gamma C+K C^{2}+2 \varepsilon n_{0}(\varepsilon) K M_{\alpha}^{\varepsilon} .
$$

Since the sequence $\left(M_{\alpha}^{\varepsilon}\right)_{\varepsilon>0}$ is uniformly bounded with respect to $\varepsilon$ by Lemma 4.1 (recall that $\alpha \leq 1+\sigma$ ), the sequence $\left(v^{\varepsilon}\right)_{\varepsilon>0}$ satisfies a uniform Lipschitz criterion on $[0, T]$. This concludes the proof of Lemma 4.2 .

Proof of Theorem 3.3.

Proof. By the Arzela-Ascoli theorem and Lemma 4.2, there exists a function $V \in \mathcal{C}([0, T])$ and a subsequence that we still denote $v^{\varepsilon}$ such that

$$
v^{\varepsilon}(t) \longrightarrow V(t) \quad \text { in } \quad \mathcal{C}([0, T])
$$

In the same way, the moment estimates of Lemma 4.1 give uniform boundedness for $\left(1+x+x^{1+\sigma}\right) u^{\varepsilon}$ in $\mathcal{M}^{1}([0, \infty))$. Pick a function $\varphi \in \mathcal{C}_{c}^{\infty}([0, \infty))$. We define

$$
\varphi_{i}^{\varepsilon}=\int_{i \varepsilon}^{(i+1) \varepsilon} \varphi(x) \mathrm{d} x,
$$

so that $\sum_{n_{0} \varepsilon}^{\infty} u_{i}^{\varepsilon} \varphi_{i}^{\varepsilon}=\int_{0}^{\infty} u^{\varepsilon}(t, x) \varphi(x) \mathrm{d} x$, and also for $y \in[j \varepsilon,(j+1) \varepsilon[$,

$$
\int_{0}^{y} k^{\varepsilon}(x, y) \varphi(x) d x=\int_{0}^{j \varepsilon} k^{\varepsilon}(x, j \varepsilon) \varphi(x) d x=\sum_{i=0}^{j} k_{i, j} \frac{\varphi_{i}^{\varepsilon}}{\varepsilon} .
$$

Thanks to the moment estimates of Lemma 4.1, and using (4.1), we have

$\left|\frac{\mathrm{d}}{\mathrm{d} t} \int_{0}^{\infty} u^{\varepsilon}(t, x) \varphi(x) \mathrm{d} x\right| \leq C\left(\|\varphi\|_{\infty}+\left\|\varphi^{\prime}\right\|_{\infty}\right) \quad$ and $\quad\left|\int_{0}^{\infty} u^{\varepsilon}(t, x) \varphi(x) \mathrm{d} x\right| \leq C\|\varphi\|_{\infty}$

for some constant $C$ depending only on $K, M_{0}, M_{1+\sigma}, \rho^{0}, \lambda, T$. Therefore, for any function $\varphi \in \mathcal{C}_{c}^{\infty}([0, \infty))$, the integral $\int u^{\varepsilon}(\cdot, x) \varphi(x) \mathrm{d} x$ is equibounded and equicontinuous. Using a density argument, we can extend this property to $\varphi \in \mathcal{C}_{0}([0, \infty))$, the space of continuous functions on $[0, \infty)$ that tend to 0 at infinity. This means that $\left(\int_{0}^{\infty} u^{\varepsilon}(., x) \varphi(x) \mathrm{d} x\right)$, belongs to a compact set of $\mathcal{C}(0, T)$. As in [6], by using the separability of $\mathcal{C}_{0}([0, \infty))$ and the Cantor diagonal process, we can extract a subsequence $u^{\varepsilon_{n}}$ and $U \in \mathcal{C}\left([0, T] ; \mathcal{M}^{1}([0, \infty))\right.$ - weak $\left.-\star\right)$, such that the following convergence

$$
\int_{0}^{\infty} u^{\varepsilon_{n}}(t, x) \varphi(x) \mathrm{d} x \rightarrow \int_{0}^{\infty} U(t, \mathrm{~d} x) \varphi(x)
$$

as $\varepsilon_{n} \rightarrow 0$, holds uniformly on $[0, T]$, for any $\varphi \in \mathcal{C}_{0}([0, \infty))$. As $u^{\varepsilon}(t, x)=0$ for $x \leq$ $\varepsilon n_{0}(\varepsilon)$, we check that $U(t,$.$) has its support in \left[x_{0}, \infty[\right.$. It remains to prove that $(U, V)$ satisfies (2.10) (2.11).

Let $\varphi$ be a smooth function supported in $[\delta, M]$ with $x_{0}<\delta<M<+\infty$, choosing $\varepsilon n_{0}(\varepsilon)+2 \varepsilon<\delta$ (what is possible due to (3.6)). By using Lemma 3.1 and Lemma A.1, we check that, for a suitable subsequence, one has

$$
\begin{aligned}
& \int_{0}^{\infty} \mu^{\varepsilon_{n}}(x) u^{\varepsilon_{n}}(t, x) \varphi(x) \mathrm{d} x \underset{\varepsilon_{n} \rightarrow 0}{\longrightarrow} \int_{0}^{\infty} \mu(x) U(t, \mathrm{~d} x) \varphi(x), \\
& \int_{0}^{\infty} \beta^{\varepsilon_{n}}(x) u^{\varepsilon_{n}}(t, x) \varphi(x) \mathrm{d} x \underset{\varepsilon_{n} \rightarrow 0}{\longrightarrow} \int_{0}^{\infty} \beta(x) U(t, \mathrm{~d} x) \varphi(x), \\
& \int_{0}^{\infty} \tau^{\varepsilon_{n}}(x) u^{\varepsilon_{n}}(t, x) \varphi(x) \mathrm{d} x \underset{\varepsilon_{n} \rightarrow 0}{\longrightarrow} \int_{0}^{\infty} \tau(x) U(t, \mathrm{~d} x) \varphi(x),
\end{aligned}
$$


uniformly on $[0, T]$. Equation (4.1) can be recast in the following integral form

$$
\begin{aligned}
& \frac{\mathrm{d}}{\mathrm{d} t} \int_{0}^{\infty} u^{\varepsilon}(t, x) \varphi(x) \mathrm{d} x \\
= & -\int_{x_{0}}^{\infty} \mu^{\varepsilon} u^{\varepsilon}(t, x) \varphi(x) \mathrm{d} x-v^{\varepsilon} \int_{0}^{\infty} \tau^{\varepsilon} u^{\varepsilon} \Delta^{\varepsilon} \varphi(x) \mathrm{d} x \\
& -\int_{0}^{\infty} \beta^{\varepsilon} u^{\varepsilon}(t, x) \varphi(x) \mathrm{d} x+2 \int_{0}^{\infty} \int_{x}^{\infty} \varphi(x) \beta^{\varepsilon}(y) u^{\varepsilon}(t, y) k^{\varepsilon}(x, y) \mathrm{d} x \mathrm{~d} y
\end{aligned}
$$

where we have defined

$$
\Delta^{\varepsilon} \varphi(x)=\frac{\varphi(x+\varepsilon)-\varphi(x)}{\varepsilon},
$$

and thereby

$$
\int_{i \varepsilon}^{(i+1) \varepsilon} \Delta^{\varepsilon} \varphi(x) \mathrm{d} x=\frac{\varphi_{i+1}^{\varepsilon}-\varphi_{i}^{\varepsilon}}{\varepsilon} .
$$

In the right hand side of (4.5), the first and third terms are treated in (4.4). Using (4.4) again and remarking that $\left|\Delta^{\varepsilon}(x)-\varphi^{\prime}(x)\right| \leq \varepsilon\left\|\varphi^{\prime \prime}\right\|_{\infty}$, we have

$$
\int_{0}^{\infty} \tau^{\varepsilon_{n}}(x) u^{\varepsilon_{n}}(t, x) \Delta^{\varepsilon_{n}} \varphi(x) \mathrm{d} x \underset{\varepsilon_{n} \rightarrow 0}{\longrightarrow} \int_{0}^{\infty} \tau(x) U(t, \mathrm{~d} x) \varphi^{\prime}(x),
$$

uniformly on $[0, T]$. Let us now study the convergence of the last term in (4.5). To this end, we use the notation $\phi$ and $\phi^{\varepsilon}$ as defined in (3.10) of Lemma 3.2 and we rewrite

$$
2 \int_{x_{0}}^{\infty} \int_{x_{0}}^{y} \varphi(x) k^{\varepsilon}(x, y) u^{\varepsilon}(t, y) \beta^{\varepsilon}(y) \mathrm{d} x \mathrm{~d} y=2 \int_{x_{0}}^{\infty} u^{\varepsilon}(t, y) \beta^{\varepsilon}(y) \phi^{\varepsilon}(y) \mathrm{d} y .
$$

Owing to (3.9) we use Lemma 3.2 which leads to

$$
\phi^{\varepsilon_{n}} \underset{\varepsilon_{n} \rightarrow 0}{\longrightarrow} \phi \quad \text { uniformly on }\left[x_{0}, R\right] \text { for any } R>0,
$$

and thus also

$$
\beta^{\varepsilon_{n}} \phi^{\varepsilon_{n}} \underset{\varepsilon_{n} \rightarrow 0}{\longrightarrow} \beta \phi \quad \text { uniformly on } \quad\left[x_{0}, R\right] \text { for any } R>0,
$$

for a suitable subsequence. Finally, we observe that $\phi^{\varepsilon_{n}}$ and therefore $\phi$ are bounded by $\|\varphi\|_{\infty}$. Thus, by using the boundedness of the higher order moments of $u^{\varepsilon}$ in Lemma 4.1 with $1+\sigma>\alpha$, we show that the fragmentation term passes to the limit (see Lemma A.1 in the Appendix). We finally arrive at

$$
\begin{aligned}
& \int_{x_{0}}^{\infty} U(t, \mathrm{~d} x) \varphi(x)-\int_{x_{0}}^{\infty} U(0, \mathrm{~d} x) \varphi(x) \\
& =-\int_{0}^{t} \int_{x_{0}}^{\infty} \mu U(t, \mathrm{~d} x) \varphi(x)-\int_{0}^{t} V(s) \int_{x_{0}}^{\infty} \tau(x) U(s, \mathrm{~d} x) \varphi^{\prime}(x) \\
& \quad-\int_{0}^{t} \int_{x_{0}}^{\infty} \beta(x) U(s, \mathrm{~d} x)(t, x) \varphi(x)+2 \int_{0}^{t} \int_{x_{0}}^{\infty} \beta(y) U(s, \mathrm{~d} y) \int_{0}^{y} \varphi(x) k(\mathrm{~d} x, y),
\end{aligned}
$$


which is the weak formulation (2.10). Moreover, (4.3) recasts as

$$
\begin{aligned}
v^{\varepsilon}(t)+\int_{0}^{\infty} e^{\varepsilon}(x) u^{\varepsilon}(t, x) \mathrm{d} x= & v^{0, \varepsilon}(t)+\int_{0}^{\infty} e^{\varepsilon}(x) u^{\varepsilon}(0, x) \mathrm{d} x \\
& +\lambda t-\gamma \int_{0}^{t} v^{\varepsilon}(s) \mathrm{d} s-\int_{0}^{t} \int_{0}^{\infty} e^{\varepsilon}(x) \mu^{\varepsilon}(x) u^{\varepsilon}(s, x) \mathrm{d} x \mathrm{~d} s,
\end{aligned}
$$

where

$$
e^{\varepsilon}(x)=\sum_{i=0}^{\infty} \varepsilon i \chi_{[i \varepsilon,(i+1) \varepsilon)}(x) .
$$

Clearly $e^{\varepsilon}(x)$ converges to $x$ uniformly. Using the moment estimate in Lemma 4.1, with $\sigma>0$, we obtain

$$
v^{\varepsilon_{n}}(t)+\int_{0}^{\infty} e^{\varepsilon_{n}}(x) u^{\varepsilon_{n}}(t, x) \mathrm{d} x \underset{\varepsilon_{n} \rightarrow 0}{\longrightarrow} V(t)+\int_{0}^{\infty} x U(t, \mathrm{~d} x)
$$

uniformly on $[0, T]$ as well as

$$
\int_{0}^{t} \int_{0}^{\infty} e^{\varepsilon_{n}}(x) \mu^{\varepsilon_{n}}(x) u^{\varepsilon_{n}}(s, x) \mathrm{d} x \mathrm{~d} s \underset{\varepsilon_{n} \rightarrow 0}{\longrightarrow} \int_{0}^{t} \int_{0}^{\infty} x \mu(x) U(s, \mathrm{~d} x) \mathrm{d} s .
$$

We refer again to Lemma A.1, or a slight adaptation of it. As $\varepsilon_{n} \rightarrow 0$ we are thus led to $(2.11)$.

\section{Proof of Theorem 3.4.}

Proof. We rewrite the rescaled ODE as

$$
\frac{d v^{\varepsilon}}{d t}=\lambda-\gamma v^{\varepsilon}-\int_{n_{0} \varepsilon}^{\infty} \tau^{\varepsilon}(x) u^{\varepsilon}(t, x) d x+2 \int_{n_{0} \varepsilon}^{\infty} \beta^{\varepsilon}(y) u^{\varepsilon}(t, y) \int_{0}^{n_{0}^{\varepsilon}} e^{\varepsilon}(x) k^{\varepsilon}(x, y) d x .
$$

Depending on the value of $x_{0}$ defined in assumption (3.6), we have to care about the last term $\left(x_{0}>0\right)$ or the next two last terms $\left(x_{0}=0\right)$. As already remarked in the proof of Lemma 4.2, in case where $x_{0}=0$, the fragmentation term can be dominated by

$$
2 \varepsilon^{2+\alpha} \sum_{i \geq n_{0}} \sum_{j<n_{0}} j k_{j, i} \beta_{i} u^{\varepsilon} \leq 2 \varepsilon n_{0}(\varepsilon) K M_{\alpha}^{\varepsilon}
$$

Hence this contribution vanishes as $\varepsilon$ goes to 0 when $\lim _{\varepsilon \rightarrow 0} \varepsilon n_{0}(\varepsilon)=x_{0}=0$. Nevertheless for case i) we still have to justify that $\int_{0}^{\infty} \tau^{\varepsilon}(x) u^{\varepsilon}(t, x) \mathrm{d} x$ passes to the limit. We obtain

$$
\int_{n_{0}\left(\varepsilon_{n}\right) \varepsilon_{n}}^{\infty} \tau^{\varepsilon_{n}} u^{\varepsilon_{n}}(t, x) \mathrm{d} x \underset{\varepsilon_{n} \rightarrow 0}{\longrightarrow} \int_{x_{0}}^{\infty} \tau U(t, \mathrm{~d} x), \quad \text { in } \quad \mathcal{C}([0, T])
$$

by using the strengthened assumption $0<\theta \leq 1$ in (3.7). Indeed, it implies that $\tau^{\varepsilon}(x)$ converges uniformly to $\tau(x)$ on any compact set $[0, R]$ while these functions do not grow faster than $x$ at infinity. We can thus use Lemma A.1 to conclude.

In the situation ii), another difficulty comes from the fragmentation term since we have to prove that

$$
\begin{aligned}
2 \int_{n_{0}\left(\varepsilon_{n}\right) \varepsilon_{n}}^{\infty} \int_{0}^{n_{0}\left(\varepsilon_{n}\right) \varepsilon_{n}} e^{\varepsilon_{n}}(x) k^{\varepsilon_{n}}(x, y) \beta^{\varepsilon_{n}}(y) u^{\varepsilon_{n}}(t, y) \mathrm{d} x \mathrm{~d} y \\
\underset{\varepsilon_{n} \rightarrow 0}{\longrightarrow} 2 \int_{x_{0}}^{\infty} \int_{0}^{x_{0}} x k(\mathrm{~d} x, y) \beta(y) U(t, \mathrm{~d} y)
\end{aligned}
$$


The stronger compactness assumptions (3.11) are basically Ascoli-type assumptions on the repartition function associated to the kernels $k^{\varepsilon}$. Denoting, in a similar manner to Appendix B:

$$
F^{\varepsilon}(x, y)=\int_{0}^{x} k^{\varepsilon}(z, y) \mathrm{d} z, \quad G^{\varepsilon}(x, y)=\int_{0}^{x} F^{\varepsilon}(z, y) \mathrm{d} z,
$$

Lemma B.6 (see Appendix B) ensures that $F^{\varepsilon} \rightarrow F$ uniformly on compact sets of $\mathbb{R}_{+} \times\left[x_{0},+\infty\right)$. We also make the remark that

$$
\begin{aligned}
\left|\int_{0}^{n_{0} \varepsilon} e^{\varepsilon}(x) k^{\varepsilon}(x, y) \mathrm{d} x-\int_{0}^{n_{0} \varepsilon} x k^{\varepsilon}(x, y) \mathrm{d} x\right| \leq \varepsilon, & \\
\int_{0}^{n_{0} \varepsilon} x k^{\varepsilon}(x, y) \mathrm{d} x & =\left[x F^{\varepsilon}(x, y)\right]_{x=0}^{x=n_{0} \varepsilon}-\int_{0}^{n_{0} \varepsilon} F^{\varepsilon}(x, y) \mathrm{d} x \\
& =\left(n_{0} \varepsilon\right) F^{\varepsilon}\left(n_{0} \varepsilon, y\right)-G^{\varepsilon}\left(n_{0} \varepsilon, y\right) .
\end{aligned}
$$

Thanks to Lemma B.6, we know that the concerned quantities are uniformly bounded and converge uniformly on compact sets, so that

$$
\int_{0}^{n_{0} \varepsilon_{n}} e^{\varepsilon_{n}}(x) k^{\varepsilon_{n}}(x, y) \mathrm{d} x \underset{\varepsilon_{n} \rightarrow 0}{\longrightarrow} \int_{0}^{x_{0}} x k(\mathrm{~d} x, y) \quad \text { uniformly on compact sets. }
$$

As before this is sufficient to prove that

$$
\begin{aligned}
2 \int_{n_{0} \varepsilon_{n}}^{\infty} \beta^{\varepsilon_{n}}(y) u^{\varepsilon_{n}}(t, y) \int_{0}^{n_{0} \varepsilon_{n}} e^{\varepsilon_{n}}(x) k^{\varepsilon_{n}}(x, y) \mathrm{d} x \mathrm{~d} y \\
\underset{\varepsilon_{n} \rightarrow 0}{\longrightarrow} 2 \int_{x_{0}}^{\infty} \beta(y) U(t, \mathrm{~d} y) \int_{0}^{x_{0}} x k(\mathrm{~d} x, y) .
\end{aligned}
$$

\section{Boundary condition for the continuous system}

The discrete system (1.1) only needs an initial condition prescribing the $u_{i}$ 's and $v$ at time $t=0$ to be well-posed, as Theorem 2.1 states. It is different for the continuous system (1.2), (1.3): a boundary condition at $x=x_{0}$ is needed when $\tau\left(x_{0}\right)>0$ (in which case the characteristics associated to the "velocity" $\tau$ are "incoming"). Even when $\tau\left(x_{0}\right)=0$, difficulties might arise when $x \mapsto \tau(x)$ is not regular enough to define the associated characteristics. However, according to the analysis of $[8,13]$, we have seen that the notion of "monomer preserving solution" appears naturally, inserting (2.9) as a constraint. It leads to the question of deciding how this condition is related to (1.2) and (1.3) and to determine the corresponding boundary condition to be used at $x=x_{0}$.

Let $(U, V)$ be a "monomer preserving" solution. In this section we do not care about the regularity requirement, and we perform several manipulations on the solution (that is assuming all the necessary integrability conditions). We suppose that the kernel $k$ splits into a Dirac mass at $x=x_{0}$ and a measure which is diffuse at $x_{0}$ :

$$
k(\mathrm{~d} x, y)=l(\mathrm{~d} x, y)+\delta_{x=x_{0}^{+}}(\mathrm{d} x) \psi^{+}(y)+\delta_{x=x_{0}^{-}}(\mathrm{d} x) \psi^{-}(y),
$$

where for any $y \geq 0, l\left(\left[x_{0}-\eta, x_{0}+\eta\right], y\right) \rightarrow 0$ as $\eta$ goes to 0 . Here we have defined $\delta_{x=x_{0}^{ \pm}}$ by

$$
\forall \phi \in \mathcal{C}_{b}\left(\left[x_{0}, \infty\right)\right), \quad<\delta_{x=x_{0}^{+}}, \phi>=\phi\left(x_{0}\right) ; \quad \forall \phi \in \mathcal{C}_{b}\left(\left(0, x_{0}\right]\right), \quad<\delta_{x=x_{0}^{+}}, \phi>=\phi\left(x_{0}\right) .
$$


Both of them are actually a Dirac mass at $x_{0}$, but we have to distinguish between $x_{0}^{+}$ and $x_{0}^{-}$because both their biological and mathematical interpretation are different. The Dirac mass at $x_{0}^{+}$means that polymers of size $x_{0}$ are formed, whereas the Dirac mass at $x_{0}^{-}$is interpreted as breakages of polymers of size $x_{0}$ going back to the monomers compartment $V$. In terms of the asymptotic process, one can think of $\delta_{x_{0}^{-}}$as the Dirac mass at $x_{0}$ produced by using information from the left, that is defined from $k^{\varepsilon}(x, y) \chi_{\left[0, n_{0} \varepsilon[\right.}(x) \mathrm{d} x$, and $\delta_{x_{0}^{+}}$as the Dirac mass at $x_{0}$ produced by $k^{\varepsilon}(x, y) \chi_{\left[n_{0} \varepsilon, y\right]}(x) \mathrm{d} x$ which relies on the information from the right of $x_{0}$. To give a simple example, the sequence with $k_{n_{0}-1, j}=\frac{1}{2}$ and the sequence with $k_{n_{0}+1, j}=$ $\frac{1}{2}$ would respectively lead to production of $\frac{1}{2} \delta_{x_{0}^{-}}$and $\frac{1}{2} \delta_{x_{0}^{+}}$. As shown below, the mathematical treatment of each is different.

The time derivative of (2.11) leads to

$$
\frac{\mathrm{d}}{\mathrm{d} t} \varrho=\frac{\mathrm{d} V}{\mathrm{~d} t}+\int_{x_{0}}^{\infty} x \frac{\partial}{\partial t} U(t, x) \mathrm{d} x=-\int_{x_{0}}^{\infty} x \mu(x) U(t, x) \mathrm{d} x+\lambda-\gamma V .
$$

On the left hand side, we can compute the derivative of the moment of $U$ by using (1.3). We obtain

$$
\begin{aligned}
\frac{\mathrm{d}}{\mathrm{d} t} \int_{x_{0}}^{\infty} x U(t, x) \mathrm{d} x= & -\int_{x_{0}}^{\infty} x \beta U \mathrm{~d} x-\int_{x_{0}}^{\infty} x \mu U \mathrm{~d} x \\
& -V \int_{x_{0}}^{\infty} x \frac{\partial}{\partial x}(\tau U) \mathrm{d} x+2 \int_{x_{0}}^{\infty} x \int_{x}^{\infty} l(\mathrm{~d} x, y) \beta(y) U(t, y) \mathrm{d} y .
\end{aligned}
$$

In this equation, since (1.3) is only written for $x>x_{0}$, only the diffuse part of the kernel $k$ appears. Integrating by parts, the convection term yields

$$
\int_{x_{0}}^{\infty} x \frac{\partial}{\partial x}(\tau U) \mathrm{d} x=-x_{0} \tau\left(x_{0}\right) U\left(t, x_{0}\right)-\int_{x_{0}}^{\infty} \tau U \mathrm{~d} x .
$$

Now we use (1.2), which gives

$$
\begin{aligned}
\frac{\mathrm{d} V}{\mathrm{~d} t}=\lambda-\gamma V-V \int_{x_{0}}^{\infty} \tau(x) U(t, x) \mathrm{d} x & +2 \int_{x=x_{0}}^{\infty} \int_{y=0}^{x_{0}} y l(\mathrm{~d} y, x) \beta(x) U(t, x) \mathrm{d} x \\
& +2 x_{0} \int_{x=x_{0}}^{\infty} \psi^{-}(x) \beta(x) U(t, x) \mathrm{d} x .
\end{aligned}
$$

We then obtain

$$
\begin{aligned}
& x_{0} V(t) \tau\left(x_{0}\right) U\left(t, x_{0}\right)-\int_{x_{0}}^{\infty} x \beta(x) U(t, x) \mathrm{d} x \\
& \quad+2 \int_{x_{0}}^{\infty} \beta(x) U(t, x)\left(\int_{0}^{x} y l(\mathrm{~d} y, x)+x_{0} \psi^{-}(x)\right) \mathrm{d} x=0 .
\end{aligned}
$$

However, (2.4) is interpreted as

$$
2 \int_{0}^{x} y l(\mathrm{~d} y, x)+2 x_{0} \chi_{\left[x_{0}, \infty\right)}(x) \psi^{-}(x)+2 x_{0} \chi_{\left(x_{0}, \infty\right)}(x) \psi^{+}(x)=x .
$$

We are thus led to the relation

$$
x_{0}\left(V(t) \tau\left(x_{0}\right) U\left(t, x_{0}\right)-2 \int_{x_{0}}^{\infty} \psi^{+}(x) \beta(x) U(t, x) \mathrm{d} x\right)=0,
$$


which suggests the boundary condition

$$
x_{0} V \tau\left(x_{0}\right) U\left(t, x_{0}\right)=2 x_{0} \int_{x_{0}}^{\infty} \psi^{+}(x) \beta(x) U(t, x) \mathrm{d} x .
$$

(Note that written in this way it makes sense also when $x_{0}=0$.)

When $x_{0}>0$, the above calculation gives solid intuitive ground to choose equation (5.1) as a boundary condition, defining the incoming flux by means of a weighted average of the solution over the size variable. In particular if the Dirac part vanishes, that is if $\psi^{+}(x)=0$, we obtain

$$
V \tau\left(x_{0}\right) U\left(t, x_{0}\right)=0,
$$

the boundary condition proposed in [10], for constant coefficient $\tau$. It is also the boundary condition used in [13].

If $x_{0}=0$, the problem is still harder, since equation (5.1) is empty. Dividing it by $x_{0}>0$ and passing formally to the limit would however give:

$$
V \tau(0) U(0)=2 \int_{0}^{\infty} \psi^{+}(x) \beta(x) U(t, x) \mathrm{d} x .
$$

Here again, it generalizes what has been proposed in [10] for $\tau$ constant and $k(x, y)=$ $\frac{1}{y} \chi_{x \leq y}$, though without any rigorous justification, and if $\psi^{+}=0$ it imposes a vanishing incoming flux.

\section{Discussion on the parameters and choice for $\varepsilon$}

The continuous model is easier to deal with than the discrete one, and thus its use is of great interest both for mathematical analysis and numerical simulations. However, as the above derivation shows, it is necessary to precisely check its range of validity. In this section, we discuss the meaning of the scaling requirements on the parameters; a full biological discussion upon their values can be found in [14]. It is based on $[17,16]$ and references therein.

6.1. Orders of magnitude. To carry out the previous scaling limit theorem, we made the following assumptions:

$$
s=\frac{\mathcal{U}}{\mathcal{V}}=\varepsilon^{2}, \quad \nu=\frac{1}{\varepsilon}, \quad \lim _{\varepsilon \rightarrow 0} \varepsilon n_{0}(\varepsilon)=x_{0}, \quad \eta=a=c=d=1 .
$$

Let us denote $i_{0}$ to be the average size of polymers. Even if there still exists much uncertainty upon its value, we can estimate that the typical size of polymers ranges between 15 and 1000, so we can write

$$
\varepsilon_{1}=\frac{1}{i_{0}} \ll 1
$$

It is also known that the "conversion rate" of PrPc is around 5 to $10 \%$ at most (depending on the experiment, on the stage of the disease, etc.); it means that the mass of proteins present in the monomeric form is much larger than the mass of proteins involved in polymers. In terms of characteristic values, it is written as

$$
\varepsilon_{2}=\frac{i_{0} \mathcal{U}}{\mathcal{V}} \ll 1
$$


Finally, we obtain

$$
\varepsilon=\sqrt{\frac{\mathcal{U}}{\mathcal{V}}}=\sqrt{\varepsilon_{1} \varepsilon_{2}} \ll 1 .
$$

Hence, it legitimates the assumption on the parameters $s$ and $\varepsilon$. Concerning the parameter $a$, we have $a=\frac{L}{\mathcal{V}} \approx \frac{2400}{500}$, which is in the order of 1 . We have only $d_{0} \leq$ $5.10^{-2}$ : this should lead us to neglect the degradation rate of polymers and simplify the equation.

For the fragmentation frequency, it is in the order of the exponential growth rate, found experimentally to be in the order of 0.1 ; in the case of Masel's articles $[17,16]$, it is supposed that $\alpha=1$, so it seems relevant (it leads to a fragmentation frequency in the order of $\varepsilon$ ). However, it has to be precisely compared to the other small parameters which are given by the typical size $i_{0}$ and the conversion rate to justify the approximation. Moreover, the assumption of a linear fragmentation kernel $\beta$ has to be confrounted to experiments.

Concerning the aggregation rate $\mathcal{T}$ and its related parameter $\nu=\tau V$, as shown in [14] in most cases we have $\frac{1}{\nu}$ in the range of $[0.01,0.1]$, so it seems justified to suppose it small; what has to be explored is its link with the other previously seen small parameters.

6.2. Discussion on the fragmentation rates $k_{i, j}$. To illustrate the central importance of a good estimate of the orders of magnitude, we exhibit here a case where the limit is not the continuous system (1.2), (1.3), but another one. Our calculation is formal, but a complete proof should be deduced from what preceeds and from [6].

Let us take, instead of $b=\varepsilon^{\alpha}$, the following scaling

$$
b=\varepsilon^{\alpha-1},
$$

and suppose also that the fragmentation kernel verifies

$$
\begin{array}{ll}
k_{1, i}=k_{i-1, i}=\frac{1}{2}\left(1-\varepsilon r_{i}\right), & k_{i, j}=\varepsilon k_{i, j}^{0} r_{j}, \quad 2 \leq i \leq j-2, \\
\sum_{i=2}^{j-2} k_{i, j}^{0}=1, & k_{i, j}^{0}=k_{j-i, j}^{0} .
\end{array}
$$

It means that the polymers are much more likely to break at their ends than in the middle of their chain. The rescaled equation (3.5) are replaced by the following ones

$$
\left\{\begin{array}{l}
\frac{\mathrm{d} v}{\mathrm{~d} t}=\lambda-\gamma v-\varepsilon^{\theta+1} v \sum \tau_{i} u_{i}+2 \varepsilon^{1+\alpha} \sum_{i \geq n_{0}} \sum_{j<n_{0}} j k_{j, i} \beta_{i} u_{i}, \\
\frac{\mathrm{d} u_{i}}{\mathrm{~d} t}=-\varepsilon^{m} \mu_{i} u_{i}-\varepsilon^{\alpha-1} \beta_{i} u_{i}-\varepsilon^{\theta-1} v\left(\tau_{i} u_{i}-\tau_{i-1} u_{i-1}\right)+2 \varepsilon^{\alpha-1} \sum_{j>i} \beta_{j} k_{i, j} u_{j}, \\
\text { for } i \geq n_{0} .
\end{array}\right.
$$

The only changes in equation (6.1) compared to (3.5) are the fragmentation terms. In order to make their limits clearly appear, we rearrange them as follows. First, in 
the equation for $v$, we write

$$
\begin{aligned}
2 \varepsilon^{1+\alpha} \sum_{i \geq n_{0}} \sum_{j<n_{0}} j k_{j, i} \beta_{i} u_{i}= & \varepsilon^{1+\alpha} \sum_{i \geq n_{0}} \beta_{i} u_{i}+\varepsilon^{1+\alpha}\left(n_{0}-1\right) \beta_{n_{0}} u_{n_{0}} \\
& -\varepsilon^{2+\alpha} \sum_{i \geq n_{0}} r_{i} \beta_{i} u_{i}-\varepsilon^{2+\alpha}\left(n_{0}-1\right) r_{n_{0}} \beta_{n_{0}} u_{n_{0}} \\
& +2 \varepsilon^{2+\alpha} \sum_{i \geq n_{0}} \sum_{j=2}^{\min \left(n_{0}-1, i-2\right)} j k_{j, i}^{0} r_{i} \beta_{i} u_{i} .
\end{aligned}
$$

All the terms vanish when $\varepsilon$ tends to zero, except the first one, similar to the aggregation term $\varepsilon^{\theta+1} v \sum \tau_{i} u_{i}$, and the last one, similar to the usual fragmentation term in equation (3.5), where $r \beta$ replaces $\beta$ and $k^{0}$ replaces $k$. We now turn to the equation for $u_{i}$, and we obtain

$$
\begin{aligned}
-\varepsilon^{\alpha-1} \beta_{i} u_{i}+2 \varepsilon^{\alpha-1} \sum_{j>i} \beta_{j} k_{i, j} u_{j}= & -\varepsilon^{\alpha-1} \beta_{i} u_{i}+\varepsilon^{\alpha-1} \beta_{i+1} u_{i+1}-\varepsilon^{\alpha} r_{i+1} \beta_{i+1} u_{i+1} \\
& +2 \varepsilon^{\alpha} \sum_{j>i+1} k_{i, j}^{0} r_{j} \beta_{j} u_{j} .
\end{aligned}
$$

The first two terms can be treated as a derivative, like the aggregation term $-\varepsilon^{\theta-1} v\left(\tau_{i} u_{i}-\tau_{i-1} u_{i-1}\right)$; the last two ones are similar to the usual fragmentation term, where $r \beta$ replaces $\beta$ and $k^{0}$ replaces $k$.

Hence, under Assumptions (3.9) on $k_{i, j}^{0}$ and (3.8) on $r_{j}$ (with a constant as an upper bound, so that its limit $r$ should be in $\mathcal{C}_{b}$ ) and $\beta_{j}$, with $\alpha-1 \leq 1+\sigma$, denoting respectively $k^{0}, r$, and $\beta$ their limits in the sense of Lemma 3.1 and Lemma 3.2, the limit system is

$$
\left\{\begin{aligned}
\frac{\mathrm{d} v}{\mathrm{~d} t}=\lambda-\gamma v- & v \int_{x_{0}}^{\infty} \tau(x) U(t, x) \mathrm{d} x \\
& +\int_{x_{0}}^{\infty} \beta(x) U(t, x) \mathrm{d} x+2 \int_{x=x_{0}}^{\infty} \int_{y=0}^{x_{0}} y k^{0}(y, x) r(x) \beta(x) U(t, x) \mathrm{d} y \mathrm{~d} x \\
\frac{\partial u}{\partial t}=-\mu(x) U(t, x)-r(x) \beta(x) U(t, x)-v \frac{\partial}{\partial x}(\tau U) & \\
& +\frac{\partial}{\partial x}(\beta u)+2 \int_{x}^{\infty} r(y) k^{0}(x, y) \beta(y) U(t, y) \mathrm{d} y .
\end{aligned}\right.
$$

Notice also that (6.2) includes the case of "renewal" type equations (refer to [18] for instance), meaning that the ends of the polymers are more likely to break. A relevant case corresponding to the above setting consists in assuming

$$
\begin{aligned}
& k_{i-n_{0}, i}^{0}=k_{n_{0}, i}^{0}=\frac{m_{i}}{2}, \quad k_{i, j}^{0}=\tilde{k}_{i, j}\left(1-m_{j}\right) \quad \text { for } i \notin\left\{n_{0}, j-n_{0}\right\}, \\
& \sum_{i=2}^{j-2} \tilde{k}_{i, j}=1, \quad \tilde{k}_{i, j}=O\left(\frac{1}{j}\right),
\end{aligned}
$$

with $\left(m_{i}\right)$ satisfying the assumptions of Lemma 3.1 and $\tilde{k}_{i, j}$ satisfying Assumption (3.9). We also define $m(y)$ as the limit of $m^{\varepsilon}$ defined as in Lemma 3.1. Then equation (6.2) remains valid, and as in section 5 , the kernel $k^{0}$ splits into a Dirac mass at $x=x_{0}$ and a measure which is diffuse at $x_{0}$ :

$$
k^{0}(\mathrm{~d} x, y)=\tilde{k}(\mathrm{~d} x, y)(1-m(y))+\delta_{x=x_{0}^{+}}(\mathrm{d} x) m(y) .
$$


We then refer to section 5 for a formal discussion on the choice of equation (5.2) as a boundary condition. However, Theorems 3.3 and 3.4 do not apply, since such a fragmentation kernel $k$ does not satisfy Assumption (3.9).

Both of these cases mean that the ends of polymers are more likely to break. What changes is the order of magnitude of what we mean by "more likely to break": is it in the order of $\frac{1}{\varepsilon}$, in which case system (1.2), (1.3) is (formally) valid, but with a (formal) boundary condition of renewal type (5.2)? Or is the difference in the order of $\frac{1}{\varepsilon^{2}}$, in which case equation (6.2) is more likely? Refer to [14] for a more complete investigation of what model should be used in what experimental context.

6.3. Discussion on the minimal size $n_{0}$. We have seen above that to have $x_{0}=0$, it suffices to make Assumption (3.6). Having also seen that the typical size $i_{0}$ is large, and that

$$
\varepsilon^{2}=\frac{1}{i_{0}} \frac{M}{m_{1} V}, \quad \frac{M}{m_{1} V} \ll 1,
$$

it is in any case valid to suppose that

$$
\frac{1}{i_{0}}=\varepsilon^{c}, \quad 0<c<2 .
$$

Hence, Assumption (3.6) can be reformulated as:

$$
n_{0} \ll i_{0}^{\frac{1}{c}} .
$$

For $c=1$, it means $n_{0} \ll i_{0}$, which is true. On the contrary, if we suppose that $x_{0}>0$, it means that $n_{0} \approx i_{0}^{\frac{1}{c}}$ : in most cases, where for instance $i_{0}=100$ or $i_{0}=1000$, it seems irrelevant.

To conclude this section (or open the debate), it seems that for each specific experiment, like PMCA protocole, in vitro or in vivo measures, or yet for the case of recombinant $\operatorname{PrP}$ (see [19]), the orders of magnitude of each parameter should be carefully estimated, in order to adapt the previous model and stick to the biological reality, which proves to be very different in in vivo, ex vivo or in vitro situations, or yet at the beginning (when there are still very few polymers) and at the end of experiments. The previous discussion illustrates this idea, and gives some possible extensions to the model we have studied.

\section{Appendix A. Compactness of the coefficients. Proof of Lemma 3.1.}

Proof. We refer to [6] for the case $\kappa=0$. Here we prove the case where $\kappa>0$. First, we show that $z^{\varepsilon}$ is close to a subsequence satisfying the requirements of the Arzela-Ascoli theorem on $[r, R]$. We define $\tilde{z}^{\varepsilon}$ by

$$
\tilde{z}^{\varepsilon}(x)=\varepsilon^{\kappa} z_{i}+\varepsilon^{\kappa} \frac{z_{i+1}-z_{i}}{\varepsilon}(x-i \varepsilon) \text { for } i \varepsilon \leq x \leq(i+1) \varepsilon .
$$

We have

$$
\begin{aligned}
\left|\tilde{z}^{\varepsilon}(x)-z^{\varepsilon}(x)\right| & =\left|\varepsilon^{\kappa} \frac{z_{i+1}-z_{i}}{\varepsilon}(x-i \varepsilon)\right|, \\
& \leq \varepsilon^{\kappa}\left|z_{i+1}-z_{i}\right|, \\
& \leq \varepsilon K(\varepsilon i)^{\kappa-1} \leq 2 \varepsilon\left(K r^{\kappa-1}+K R^{\kappa-1}\right) .
\end{aligned}
$$


Furthermore $\tilde{z}^{\varepsilon}$ has a bounded derivative since

$$
\begin{aligned}
\left|\frac{\mathrm{d} \tilde{z}^{\varepsilon}}{\mathrm{d} x}\right| & =\varepsilon^{\kappa} \frac{z_{i+1}-z_{i}}{\varepsilon}, \\
& \leq K(\varepsilon i)^{\kappa-1}, \\
& \leq K r^{\kappa-1}+K R^{\kappa-1} .
\end{aligned}
$$

Therefore, the family $\tilde{z}^{\varepsilon}$ satisfies the requirements of the Arzela-Ascoli theorem for any interval $[r, R]$ with $0<r<R<+\infty$. We can extract a subsequence converging uniformly to $z$. The limit is continuous and satisfies $z(x) \leq K x^{\kappa}$. When $\kappa>0$ the convergence extends on $[0, R]$ owing to the remark

$$
\sup _{x \in[0, r]}\left|\left(z^{\varepsilon}-z\right)(x)\right| \leq 2 K r .
$$

This concludes the proof.

During the proof of Theorem 3.3 and Theorem 3.4 we made repeated use of the following claim.

LEMMA A.1. Let $z_{n}$ converge to a continuous function $z$ uniformly on $[0, M]$ for any $0<M<\infty$, with $\left|z_{n}(x)\right| \leq K\left(1+x^{\kappa}\right)$. Let $\left(u_{n}\right)_{n \in \mathbb{N}}$ be a sequence of integrable functions which converges to $U$ weakly- $\star$ in $\mathcal{M}^{1}([0, \infty))$. Furthermore we suppose that

$$
\sup _{n \in \mathbb{N}} \int_{0}^{\infty}\left(1+x^{\ell}\right)\left|u_{n}(x)\right| \mathrm{d} x \leq C<\infty .
$$

Assuming $0 \leq \kappa<\ell$, we have

$$
\int_{0}^{\infty} z_{n}(x) u_{n}(x) \mathrm{d} x \underset{n \rightarrow \infty}{\longrightarrow} \int_{0}^{\infty} z(x) U(\mathrm{~d} x) .
$$

Proof. First we notice that since for any compactly supported continuous function we have

$$
\left|\int_{0}^{\infty}\left(1+x^{\ell}\right) u_{n}(x) \varphi(x) \mathrm{d} x\right| \leq C\|\varphi\|_{\infty}
$$

we have, from weak star convergence with $\left(1+x^{l}\right) \varphi(x)$ as a test function,

$$
\left|\int_{0}^{\infty}\left(1+x^{\ell}\right) \varphi(x) U(\mathrm{~d} x)\right| \leq C\|\varphi\|_{\infty}
$$

and therefore $\left(1+x^{\ell}\right) U(\mathrm{~d} x)$ is a signed measure with total variation

$$
\left\|\left(1+x^{\ell}\right) U(\mathrm{~d} x)\right\|_{V T}=\int_{0}^{\infty}(1+x)^{\ell}|U|(\mathrm{d} x) \leq C .
$$

We denote as usual $U(\mathrm{~d} x)=U^{+}(\mathrm{d} x)-U^{-}(\mathrm{d} x)$ and $|U|(\mathrm{d} x)=U^{+}(\mathrm{d} x)+U^{-}(\mathrm{d} x)$.

Let $\zeta \in C_{c}^{\infty}([0, \infty))$ such that $0 \leq \zeta(x) \leq 1, \zeta(x)=1$ on $[0, R], 0<R<\infty$, and 
$\operatorname{supp}(\zeta) \subset[0,2 R]$. We split

$$
\begin{aligned}
& \left|\int_{0}^{\infty} z_{n}(x) u_{n}(x) \mathrm{d} x-\int_{0}^{\infty} z(x) U(\mathrm{~d} x)\right| \\
& =\mid \int_{0}^{\infty} z_{n}(x) \zeta(x) u_{n}(x) \mathrm{d} x-\int_{0}^{\infty} z(x) \zeta(x) U(\mathrm{~d} x) \\
& \quad+\int_{0}^{\infty} z_{n}(x)(1-\zeta(x)) u_{n}(x) \mathrm{d} x-\int_{0}^{\infty} z(x)(1-\zeta(x)) U(\mathrm{~d} x) \mid \\
& \quad \leq \int_{0}^{\infty}\left|z_{n}(x)-z(x)\right| \zeta(x)\left|u_{n}(x)\right| \mathrm{d} x \\
& \quad+\left|\int_{0}^{\infty} z(x) \zeta(x) u_{n}(x) \mathrm{d} x-\int_{0}^{\infty} z(x) \zeta(x) U(\mathrm{~d} x)\right| \\
& \quad+\int_{0}^{\infty}\left|z_{n}(x)\right|(1-\zeta(x))\left|u_{n}(x)\right| \mathrm{d} x+\int_{0}^{\infty}|z(x)|(1-\zeta(x))|U|(\mathrm{d} x) .
\end{aligned}
$$

The two last integrals can be dominated by

$$
\begin{gathered}
K \sup _{y \geq R}\left(\frac{1+y^{\kappa}}{1+y^{\ell}}\right)\left(\sup _{n} \int_{0}^{\infty}\left(1+x^{\ell}\right)\left(\left|u_{n}(x)\right| \mathrm{d} x+\int_{0}^{\infty}\left(1+x^{\ell}\right)|U|(\mathrm{d} x)\right)\right. \\
\leq 2 C K \sup _{y \geq R}\left(\frac{1+y^{\kappa}}{1+y^{\ell}}\right) .
\end{gathered}
$$

Since $0 \leq \kappa<\ell$, this contribution can be made arbitrarily small by choosing $R$ large enough, uniformly with respect to $n$. Moreover, we clearly have

$$
\begin{aligned}
0 \leq \int_{0}^{\infty}\left|z_{n}(x)-z(x)\right| & \left|u_{n}(x)\right| \zeta(x) \mathrm{d} x \\
& \leq \sup _{0 \leq x \leq 2 R}\left|z_{n}(x)-z(x)\right| \sup _{m} \int_{0}^{\infty}\left|u_{m}(x)\right| \mathrm{d} x \underset{n \rightarrow \infty}{\longrightarrow} 0
\end{aligned}
$$

and of course

$$
\int_{0}^{\infty} z(x) \zeta(x) u_{n}(x) \mathrm{d} x-\int_{0}^{\infty} z(x) \zeta(x) U(\mathrm{~d} x) \underset{n \rightarrow \infty}{\longrightarrow} 0 .
$$

Combining all together these informations ends the proof.

Appendix B. Compactness of the fragmentation kernel. We look for conditions on the coefficients guaranteeing some compactness of $k^{\varepsilon}$. We use a few classical results of convergence of probability measures (see [3] for instance). Let us introduce a few notations. Given a probability-measure-valued function $y \in \mathbb{R} \mapsto k(., y) \in \mathcal{M}^{1}(\mathbb{R})$, we denote $F(., y)$ to be its repartition function: $F(x, y)=\int_{-\infty}^{x} k(s, y) \mathrm{d} s$ and $G(x, y)$ to be the function $\int_{-\infty}^{x} F(z, y) \mathrm{d} z$. We shall deduce the compactness of $k^{\varepsilon}$ from the compactness of the associated $G^{\varepsilon}$. To this end, we need several elementary statements.

Lemma B.1. Let $\left\{P^{n}, n \in \mathbb{N}\right\}$ be a family of probability measures on $\mathbb{R}$, having their support included in some interval $[a, b]$. We denote $F^{n}$ to be the repartition function of $P^{n}$, and $G^{n}$ to be the functions defined by $\int_{-\infty}^{x} F^{n}(s) \mathrm{d} s$. The following assertions are equivalent:

$$
\text { 1. } P^{n} \rightarrow P \text { weakly, i.e., } \forall f \in \mathcal{C}_{b}(\mathbb{R}), P_{n} f \rightarrow P f,
$$


2. $F^{n}(x) \rightarrow F(x)$ for all $x$ at which $F$ is continuous,

3. $G^{n} \rightarrow G$ uniformly locally.

Lemma B.2 (Conditions for $F$ ). Let $F$ be a nondecreasing function on $\mathbb{R}$. There exists a unique probability measure $P$ on $\mathbb{R}$, such that $F(x)=P(]-\infty, x])$, iff

- $F$ is rightcontinuous everywhere,

- $\lim _{x \rightarrow-\infty} F(x)=0, \lim _{x \rightarrow+\infty} F(x)=1$.

Furthermore $P$ has its support included in $[a, b]$ iff $F \equiv 0$ on $]-\infty, a[$ and $F(b)=1$.

Lemma B.3 (Conditions for $G$ ). Let $G$ be a convex function on $\mathbb{R}$. There exists a probability measure $P$ on $\mathbb{R}$, having its support included in $[a, b]$, such that $G(x)=$ $\int_{-\infty}^{x} F(s) \mathrm{d} s$, where $\left.\left.F(x)=P(]-\infty, x\right]\right)$, iff

- $G$ is increasing,

- for $x>b, G(x)=G(b)+x$,

- $G \equiv 0$ on $]-\infty, a]$.

Corollary B.4. Let $\left(G^{n}\right)_{n \in \mathbb{N}}$ a sequence satisfying the assumptions of Lemma B.3. Suppose $G^{n} \rightarrow G$ uniformly locally on $\mathbb{R}$, then $G$ also satisfy these assumptions and we have $P^{n} \rightarrow P$ weakly.

Proof. We define the function $F$ as $F(x)=\lim _{\delta \rightarrow 0^{+}} \frac{G(x+\delta)-G(x)}{\delta}$, it is then easy to check that $F$ satisfies assumptions of Lemma B.2, and $G(x)=\int_{-\infty}^{x} F(s) \mathrm{d} s$.

Proof of Lemma 3.2.

Proof. We prove the following result, which contains Lemma 3.2.

Lemma B.5. Suppose that the discrete coefficients satisfy (3.9). Then there exist a subsequence $\varepsilon_{n}$ and $k \in \mathcal{C}\left([0, \infty), \mathcal{M}_{+}^{1}([0, \infty))\right.$ - weak $\left.-\star\right)$ such that

- $k$ satisfies (2.3),(2.2) (and therefore (2.4)),

- for every $y>0, k^{\varepsilon_{n}}(., y) \rightarrow k(., y)$ in law,

- for every $\varphi \in \mathcal{C}_{c}^{\infty}([0, \infty)), \phi^{\varepsilon_{n}} \rightarrow \phi$ uniformly on $[0, R]$ for any $0<R<\infty$.

For any $y \geq 0, k^{\varepsilon}(x, y) \mathrm{d} x$ defines a probability measure on $[0, \infty)$, supported in $[0, y]$. We set $F^{\varepsilon}(x, y)=\int_{0}^{x} k^{\varepsilon}(z, y) \mathrm{d} z$ and $G^{\varepsilon}(x, y)=\int_{0}^{x} F^{\varepsilon}(z, y) \mathrm{d} z$. Let $\varphi \in C_{c}^{\infty}\left(\mathbb{R}_{+}^{*}\right)$. We start by rewriting, owing to integration by parts,

$$
\phi^{\varepsilon}(y)=\varphi(y)-\int_{0}^{y} F^{\varepsilon_{n}}(x, y) \varphi^{\prime}(x) \mathrm{d} x=\varphi(y)-G^{\varepsilon}(y, y) \varphi^{\prime}(y)+\int_{0}^{y} G^{\varepsilon}(x, y) \varphi^{\prime \prime}(x) \mathrm{d} x,
$$

where we used the fact that $F^{\varepsilon}(y, y)=\int_{0}^{y} k^{\varepsilon}(z, y) \mathrm{d} z=1$. The proof is based on the following argument: $G^{\varepsilon}$ is close to a $\tilde{G}^{\varepsilon}$ which satisfies the assumptions of the ArzelaAscoli theorem. Given $x, y \geq 0$ and $\varepsilon>0, i, j$ denote the integers satisfying $x \in[i \varepsilon,(i+$ 1) $\varepsilon[, y \in[j \varepsilon,(j+1) \varepsilon[$ and a short computation leads to

$$
\begin{aligned}
& F^{\varepsilon}(x, j \varepsilon)=S_{i, j}+\frac{x-i \varepsilon}{\varepsilon} k_{i, j}, \\
& G^{\varepsilon}(x, j \varepsilon)=\varepsilon \sum_{p=0}^{i-1} S_{p, j}+(x-i \varepsilon) S_{i, j}+\frac{\varepsilon}{2} S_{i, j}+\frac{(x-i \varepsilon)^{2}}{2 \varepsilon} k_{i, j},
\end{aligned}
$$

where

$$
S_{i, j}=\sum_{r=0}^{i-1} k_{r, j} .
$$


We define

$$
\tilde{k}^{\varepsilon}(x, y)=\frac{(j+1) \varepsilon-y}{\varepsilon} k^{\varepsilon}(x, j \varepsilon)+\frac{y-j \varepsilon}{\varepsilon} k^{\varepsilon}(x,(j+1) \varepsilon)
$$

and we have

$$
\tilde{G}^{\varepsilon}(x, y)=\frac{(j+1) \varepsilon-y}{\varepsilon} G^{\varepsilon}(x, j \varepsilon)+\frac{y-j \varepsilon}{\varepsilon} G^{\varepsilon}(x,(j+1) \varepsilon) .
$$

Observe that

$$
\begin{aligned}
\left|\tilde{G}^{\varepsilon}(x, y)-G^{\varepsilon}(x, y)\right| & =\frac{y-j \varepsilon}{\varepsilon}\left|G^{\varepsilon}(x,(j+1) \varepsilon)-G^{\varepsilon}(x, j \varepsilon)\right| \\
\leq \mid \varepsilon \sum_{p=0}^{i-1}\left(S_{p, j+1}-S_{p, j}\right)+(x-i \varepsilon)\left(S_{i, j+1}-S_{i, j}\right)+\frac{\varepsilon}{2}\left(S_{i, j+1}-S_{i, j}\right) & +\frac{(x-i \varepsilon)^{2}}{2 \varepsilon}\left(k_{i, j+1}-k_{i, j}\right) \mid .
\end{aligned}
$$

Due to (2.3), we have $0 \leq k_{i, j} \leq 1$ and thus $\left|k_{i, j+1}-k_{i, j}\right| \leq 1$. Similarly $0 \leq S_{i, j} \leq 1$ and $\left|S_{i, j+1}-S_{i, j}\right| \leq 1$. Hence, since (3.9) can also be written

$$
\left|\sum_{p=0}^{i-1} S_{p, j+1}-S_{p, j}\right| \leq K,
$$

it allows us to obtain

$$
\left|\tilde{G}^{\varepsilon}(x, y)-G^{\varepsilon}(x, y)\right| \leq \varepsilon(K+1+1 / 2+1 / 2)=\varepsilon(K+2) .
$$

We also deduce that

$$
\left|\partial_{y} \tilde{G}^{\varepsilon}(x, y)\right|=\frac{\left|G^{\varepsilon}(x, j \varepsilon)-G^{\varepsilon}(x,(j+1) \varepsilon)\right|}{\varepsilon} \leq K+2
$$

while

$$
\left|\partial_{x} \tilde{G}^{\varepsilon}(x, y)\right| \leq 1
$$

Moreover, we have

$$
\left|\tilde{G}^{\varepsilon}(x, y)\right| \leq 2 \varepsilon(i+2)
$$

which is bounded uniformly with respect to $\varepsilon$ and $0 \leq x, y \leq R<\infty$. As a consequence of the Arzela-Ascoli theorem we deduce that, for a subsequence, $G^{\varepsilon_{n}}$ converges uniformly to a continuous function $G(x, y)$ on $[0, R] \times[0, R]$ for any $0<R<\infty$. It follows that

$$
\phi^{\varepsilon_{n}}(y) \underset{\varepsilon_{n} \rightarrow \infty}{\longrightarrow} \phi(y)=\varphi(y)-G(y, y) \varphi^{\prime}(y)+\int_{0}^{y} G(x, y) \varphi^{\prime \prime}(x) \mathrm{d} x
$$

uniformly on $[0, R]$. We conclude by applying Lemma B.3 to the function $x \mapsto G(x, y)$, with $y \geq 0$ fixed.

Lemma B.6. Suppose that the discrete coefficients satisfy (3.11). Then $F^{\varepsilon}$ and $G^{\varepsilon}$ are uniformly bounded and converge (up to a subsequence) uniformly on compact sets. 
Proof. Assumption (3.11) rewrites

$$
\left|S_{i, j+1}-S_{i, j}\right| \leq \frac{K}{j}, \quad k_{i, j} \leq \frac{K}{j},
$$

where $S_{i j}$ is defined as in (B.1). So, with the same notation for $\tilde{F}^{\varepsilon}$ as for $\tilde{G}^{\varepsilon}$ and $\tilde{k}^{\varepsilon}$, we obtain

$$
\left|F^{\varepsilon}(x, y)-\tilde{F}^{\varepsilon}(x, y)\right|=\frac{y-j \varepsilon}{\varepsilon}\left|F^{\varepsilon}(x,(j+1) \varepsilon)-F^{\varepsilon}(x, j \varepsilon)\right| \leq \frac{2 K}{j} \leq \frac{3 K}{y} \varepsilon .
$$

Similar considerations yield

$$
\left|\partial_{x} \tilde{F}^{\varepsilon}\right| \leq \frac{(j+1) \varepsilon-y}{\varepsilon} k_{i j}+\frac{y-j \varepsilon}{\varepsilon} k_{i, j+1} \varepsilon \leq \frac{2 K}{j \varepsilon} \leq \frac{2 K}{n_{0} \varepsilon} \leq \frac{2 K}{x_{0}},
$$

where we have used Assumption (3.6), restricting the discussion to the case $x_{0}>0$ (see case ii) in Theorem 3.4) and

$$
\left|\partial_{y} \tilde{F}^{\varepsilon}\right| \leq \frac{1}{\varepsilon}\left|F^{\varepsilon}(x,(j+1) \varepsilon)-F^{\varepsilon}(x, j \varepsilon)\right| \leq \frac{3 K}{y},
$$

which leads to Ascoli assumptions and therefore the suitable compactness.

With such assumptions, we can take into account any $k$ of the form $k(x, y) \mathrm{d} x=$ $\frac{1}{y} k_{0}(x / y) \mathrm{d} x$, including Dirac mass. If we consider such a distribution on $[0,1]$ (taken symmetric), then we can define $k_{i, j}$ as

$$
\begin{aligned}
k_{i, j}=k_{0} & (] \frac{i-1}{j-1}, \frac{i}{j-1}[)+\frac{1}{2} k_{0}\left(\left\{\frac{i-1}{j-1}\right\}\right) \\
& +\frac{1}{2} k_{0}\left(\left\{\frac{i}{j-1}\right\}\right)+\frac{1}{2} k_{0}(\{0\}) \delta_{i}^{1}+\frac{1}{2} k_{0}(\{0\}) \delta_{i}^{j-1},
\end{aligned}
$$

with $\delta_{i}^{j}$ the Kronecker symbol. With these notations, we have for $p \geq j-2$,

$$
S_{p, j}=\sum_{i=0}^{p} k_{i, j}=k_{0}\left(\left[0, \frac{p}{j-1}[)+\frac{1}{2} k_{0}\left(\left\{\frac{p}{j-1}\right\}\right)\right.\right.
$$

and $S_{j-1, j}=S_{j, j}=1$, which leads to

$$
\begin{aligned}
& S_{p, j+1}-S_{p, j}=k_{0}\left(\left[\frac{p}{j}, \frac{p}{j-1}[)+\frac{1}{2} k_{0}\left(\left\{\frac{p}{j}\right\}\right)-\frac{1}{2} k_{0}\left(\left\{\frac{p}{j-1}\right\}\right), \quad \text { if } p<j-1,\right.\right. \\
& \left.\left.S_{j-1, j+1}-S_{j-1, j}=-k_{0}(\rceil \frac{j-1}{j}, 1\right]\right)-\frac{1}{2} k_{0}\left(\left\{\frac{j-1}{j}\right\}\right), \quad S_{j, j+1}-S_{j, j}=0,
\end{aligned}
$$

as $0 \leq i \leq j$, we have for any $p \leq i$,

$$
\frac{p-1}{j-1} \leq \frac{p}{j}
$$

the intervals $\left[\frac{p}{j}, \frac{p}{j-1}\left[\right.\right.$ and $\left[\frac{p-1}{j}, \frac{p-1}{j-1}[\right.$ are disjoint. This leads to

$$
\left|\sum_{p=0}^{i} S_{p, j+1}-S_{p, j}\right| \leq k_{0}\left(\bigcup _ { p = 0 } ^ { i } \left[\frac{p}{j}, \frac{p}{j-1}[)+\frac{1}{2} k_{0}\left(\bigcup_{p=0}^{i}\left\{\frac{p}{j}\right\}\right)+\frac{1}{2} k_{0}\left(\bigcup_{p=0}^{i}\left\{\frac{p}{j-1}\right\}\right) \leq 2\right.\right.
$$


which gives the criterion (3.9). The limit is then obviously given by $k(x, y) d x=$ $\frac{1}{y} k_{0}(x / y) d x$.

Appendix C. Discrete system. Here we briefly discuss the existence theorem for the discrete system. It is mainly an adaptation of Theorem 5.1 in [12]. We define the truncated system. Let $N>n_{0}$, consider the system

$$
\left\{\begin{array}{l}
\frac{\mathrm{d} v}{\mathrm{~d} t}=\lambda-\gamma v-v \sum_{i=n_{0}}^{N-1} \tau_{i} u_{i}+2 \sum_{j=n_{0}}^{N} \sum_{i<n_{0}} i k_{i, j} \beta_{j} u_{j}, \\
\frac{\mathrm{d} u_{n_{0}}}{\mathrm{~d} t}=-\mu_{n_{0}} u_{n_{0}}-\beta_{n_{0}} u_{n_{0}}-v \tau_{n_{0}}+2 \sum_{j=i+1}^{N} \beta_{j} k_{n_{0}, j} u_{j}, \\
\frac{\mathrm{d} u_{i}}{\mathrm{~d} t}=-\mu_{i} u_{i}-\beta_{i} u_{i}-v\left(\tau_{i} u_{i}-\tau_{i-1} u_{i-1}\right)+2 \sum_{j=i+1}^{N} \beta_{j} k_{i, j} u_{j}, \quad \text { for } n_{0}<i<N, \\
\frac{\mathrm{d} u_{N}}{\mathrm{~d} t}=-\mu_{N} u_{N}-\beta_{N} u_{N}+v \tau_{N-1} u_{N-1} .
\end{array}\right.
$$

Existence, uniqueness and nonnegativity are immediate, we have immediately the weak formulation

$$
\begin{aligned}
\frac{\mathrm{d}}{\mathrm{d} t}\left(v(t) \psi+\sum_{i=n_{0}}^{N} u_{i} \varphi_{i}\right)= & \lambda \psi-\gamma v \psi-v \sum_{i=n_{0}}^{N} \mu_{i} u_{i} \varphi_{i}+v \sum_{i=n_{0}}^{N-1} \tau_{i} u_{i}\left(\varphi_{i+1}-\varphi_{i}-\psi\right) \\
& +2 \sum_{j=n_{0}+1}^{N} \sum_{i=n_{0}}^{j-1} i k_{i, j} \beta_{j} u_{j}\left(\frac{\varphi_{i}}{i}-\frac{\varphi_{j}}{j}\right) \\
& +2 \sum_{j=n_{0}}^{N} \sum_{i=1}^{n_{0}-1} i k_{i, j} \beta_{j} u_{j}\left(\psi-\frac{\varphi_{j}}{j}\right) .
\end{aligned}
$$

Let us denote $U^{N}$ the infinite sequence of functions defined by $U_{i}^{N}=u_{i}^{n}$ if $n_{0} \leq i \leq N$, $U_{i}^{N}=0$ otherwise. The weak formulation gives moment estimates (and the moment estimates done in section 4 can then be thought as uniform bounds on truncated systems). This model has the property of propagating moments.

With this type of initial condition, the proof of existence is based on the Ascoli theorem for the continuous functions $U_{i}^{N}$. Thanks all the moments controlled on the initial data and the nice property of propagation of moments, we have bounds on the derivative of $v^{N}, U_{i}^{N}$ and therefore, we can extract convergent subsequence. The limit satisfies the equation in an integral form (see [1] for a definition). For proving uniqueness, the procedure exposed in $[1,12]$ applies, with a small modification due to death rates (the condition on the moment of order $1+m$ for the initial data insures the convergence of $\left.\sum i \mu_{i} u_{i}\right)$.

Acknowledgement. We thank Pavel Dubovski for kind comments and suggestions about this work, and Natacha Lenuzza for fruitful discussions and help about orders of magnitude of the parameters. Part of this work has been supported by the ANR grant TOPPAZ. 


\section{REFERENCES}

[1] J.M. Ball and J. Carr, The discrete coagulation-fragmentation equations: existence, uniqueness, and density conservation, J. Statist. Phys., 61(1-2), 203-234, 1990. MR1084278 (92h:82086)

[2] J.M. Ball, J. Carr and O. Penrose, The Becker-Döring cluster equations: basic properties and asymptotic behaviour of solutions, Commun. Math. Phys., 104(4), 657-692, 1986. MR841675 (87i:82116).

[3] P. Billingsley, Convergence of Probability Measures, second ed., Wiley Series in Probability and Statistics: Probability and Statistics, John Wiley \& Sons Inc., New York, 1999.

[4] V. Calvez, N. Lenuzza, M Doumic, J.P. Deslys, F. Mouthon and B. Perthame, Prion dynamic with size dependency - strain phenomena, J. Biol. Dyn., in press, 2008.

[5] V. Calvez, N. Lenuzza, D. Oelz, J.P. Deslys, P. Laurent, F. Mouthon and B. Perthame, Size distribution dependence of prion aggregates infectivity, Math. Biosci., 1, 88-99, 2009.

[6] J.F. Collet, T. Goudon, F. Poupaud and A. Vasseur, The Becker-Döring system and its Lifshitz-Slyozov limit, SIAM J. Appl. Math., 62(5), 1488-1500, 2002.

[7] F.P. Da Costa, Existence and uniqueness of density conserving solutions to the coagulationfragmentation equations with strong fragmentation, J. Math. Anal. Appl., 192, 892-914, 1995.

[8] P. Dubovskii and I. Stewart, Existence, uniqueness and mass-conservation for the coagulationfragmentation equations, Math. Meth. Appl. Sci., 19, 571-591, 1996.

[9] M. Eigen, Prionics or the kinetic basis of prion diseases, Biophys. Chem., 63, A1-A18, 1996.

[10] M.L. Greer, L. Pujo-Menjouet and G.F. Webb, A mathematical analysis of the dynamics of prion proliferation, J. Theor. Biol., 242(3), 598-606, 2006.

[11] P. Laurençot and S. Mischler, From the discrete to the continuous coagulation-fragmentation equations, Proceedings of the Royal Society of Edinburgh: Section A Mathematics, 132(05), 1219-1248, 2007.

[12] P. Laurençot, The discrete coagulation equations with multiple fragmentation, Proc. Edinb. Math. Soc., 45(2), 1, 67-82, 2002. MR1884603 (2003b:82036).

[13] P. Laurencot and C. Walker, Well-posedness for a model of prion proliferation dynamics, Journal of Evolution Equations, 7(2), 241-264, 2007.

[14] N. Lenuzza, In Preparation, Ph.D. thesis, Paris, 2009.

[15] P. Malliavin, Intégration et Probabilités. Analyse de Fourier et Analyse Spectrale, Masson, Paris, 1982, Collection: Maîtrise de Mathématiques Pures.

[16] J. Masel, N. Genoud and A. Aguzzi, Efficient inhibition of prion replication by PrPFc2 suggests that the prion is a PrPSc oligomer, J. Mol. Biol., 345, 1243-1251, 2005.

[17] J. Masel, V.A. Jansen and M.A. Nowak, Quantifying the kinetic parameters of prion replication, Biophys. Chem., 77(2-3), 139-152, 1999.

[18] B. Perthame, Transport Equations in Biology, Frontiers in Mathematics, Birkhäuser Verlag, Basel, 2007. MR2270822 (2007j:35004).

[19] H. Rezaei, Prion protein oligomerization, Current in Alzheimer Research, 5, 572-578, 2008.

[20] J.R. Silveira, G.J. Raymond, A.G. Hughson, R.E. Race, L. Sim, V.S.F. Hayes and B. Caughey, The most infectious prion protein particles, Nature, 437, 257-261, 2005. 